UNIVERSIDADE FEDERAL DO MARANHÃO

CENTRO DE CIÊNCIAS EXATAS E TECNOLOGIA

DEPARTAMENTO DE MATEMÁTICA

PROGRAMA DE MESTRADO PROFISSIONAL

EM MATEMÁTICA EM REDE NACIONAL - PROFMAT

Rubens Lopes Netto

Uma aplicação do Teorema do Valor Médio

São Luís - MA 
Rubens Lopes Netto

\section{Uma aplicação do Teorema do Valor Médio}

Dissertação apresentada ao Programa de Mestrado Profissional em Matemática em Rede Nacional da Universidade Federal do Maranhão como requisito parcial para a obtenção do grau de Mestre em Matemática.

Orientador: Prof. Dr. Arlane Manoel Silva Vieira

São Luís - MA 
Ficha gerada por meio do SIGAA/Biblioteca com dados fornecidos pelo(a) autor(a). Núcleo Integrado de Bibliotecas/UFMA

Netto, Rubens Lopes

13pt Uma aplicação do Teorema do Valor Médio / Rubens Lopes. . - 2017

$48 \mathrm{p.}$

13pt Orientador(a): Arlane Manoel da Silva.

13pt Dissertação (Mestrado) - Programa de Pós-graduação em Rede - Matemática em Rede Nacional/ccet, Universidade Federal do Maranhão, São Luís, 2017.

13pt 1. Introdução 2. Fundamentação Teórica 3. O Teorema do Valor Extremo 4. O Teorema do Valor Intermediário 5. O Teorema de Rolle 6. Uma aplicação particular do Teorema de Rolle 7. O Teorema do Valor Médio 8. Uma aplicação particular do Teorema do Valor Médio 9. Aplicação do Teorema 8.1.1 no Ensino Médio 10. Considerações finais. I. Vieira, Arlane Manoel S. II. Título. 
Rubens Lopes Netto

\section{Uma aplicação do Teorema do Valor Médio}

Dissertação apresentada ao PROFMAT/ Universidade Federal do Maranhão como requisito parcial para a obtenção do grau de Mestre em Matemática.

Aprovado em 24/04/2017

BANCA EXAMINADORA

Prof. Dr. Arlane Manoel Silva Vieira

Prof. Dr. Jairo Santos da Silva

Prof. Dr. Giovane Ferreira Silva 
À minha amada família! 


\section{AGRADECIMENTOS}

Primeiramente a Deus por ter me abençoado com a oportunidade de dar mais este passo em minha vida estudantil e profissional.

Ao meu orientador, o professor Arlane Manoel Silva Vieira, por toda a ajuda e dedicação durante todo o trabalho.

Ao PROFMAT pela oportunidade de me aprofundar mais em minha área de formação e me capacitar ainda mais para o exercício de minha profissão.

À minha família por todo o apoio que me foi dado durante todo o curso e pela compreensão que tiveram por conta do tempo e da dedicação que tive que dispor para este curso.

Aos meus colegas de turma que tornaram-se verdadeiros amigos, ao longo deste curso, estando sempre unidos para que todos chegassem ao final com a conquista do título. 
"Até aqui nos ajudou o Senhor."

1 Samuel 7:12 


\section{RESUMO}

Nesta dissertação apresentamos uma aplicação do Teorema do Valor Médio que consiste em sua restrição a um caso particular. Este resultado se aplica às funções reais, contínuas e deriváveis em um dado intervalo.

Geometricamente esse resultado diz que, dados uma função $f$ contínua em $[a, b]$ e derivável em $(a, b)$ e dois pontos $P(a, f(a))$ e $Q(b, f(b))$ do gráfico de $f$, existe um ponto $(c, f(c))$ entre os pontos $A$ e $B$ onde a reta tangente à curva possui inclinação igual ao coeficiente angular da reta suporte da corda que une os pontos $P(a, f(a))$ e $Q(b, f(b))$ adicionado a um múltiplo da distância vertical entre o ponto $(c, f(c))$ do gráfico de $f$ e o ponto correspondente na reta secante $\stackrel{P Q}{ }$.

Palavras-chave: Números reais, intervalos, funções, continuidade, derivadas, tangentes. 


\section{ABSTRACT}

In this work we will present a new theorem that consists of the restriction of the Theorem of the Average Value to a particular case. This theorem applies to real, continuous, and derivable functions in a given interval. Its application allows us to calculate a determinated point on a curve where the straight line tangent to this curve has a specific inclination.

As this theorem is related to the concepts of inclination, tangent and secant, this one can be applied in the High School to solve problems of Analytical Geometry that involve such concepts, as we will see in some examples given.

Keywords: Real numbers, intervals, functions, continuity, derivatives, tangents. 


\section{SUMÁRIO}

1 INTRODUÇÃO

2 FUNDAMENTAÇÃO TEÓRICA 10

2.1 Números Reais . . . . . . . . . . . . . . . . . . . . 10

2.1.1 Supremo e ínfimo . . . . . . . . . . . . . . . . . . . . . 10

2.1 .2 Intervalos . . . . . . . . . . . . . . . . . 12

2.1.3 Desigualdade triangular . . . . . . . . . . . . . . 15

2.2 Limites de Funções . . . . . . . . . . . . . . . . . . . . . . . . . . . . 16

2.2.1 Propriedades dos limites . . . . . . . . . . . . . . 17

2.3 Continuidade . . . . . . . . . . . . . . . . . . . . . . . . 19

2.4 Derivada . . . . . . . . . . . . . . . . . . 21

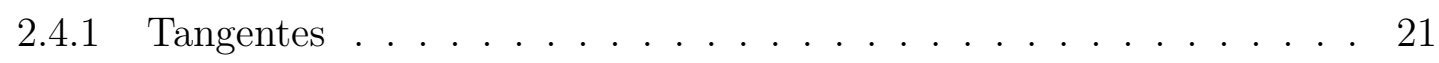

2.4.2 Equação da reta tangente . . . . . . . . . . . . . . . . 22

2.4.3 A derivada de uma função em um ponto . . . . . . . . . . . . . 22

2.4.4 Derivadas laterais . . . . . . . . . . . . . . . 23

2.4.5 Continuidade de funções deriváveis . . . . . . . . . . . . . 23

2.4.6 Regras de derivação . . . . . . . . . . . . . . . . 24

3 CONSEQUÊNCIAS DA CONTINUIDADE 26

3.1 Valores Extremos . . . . . . . . . . . . . . . . 26

3.2 O Teorema do Valor Extremo . . . . . . . . . . . . . 27

3.3 O Teorema do Valor Intermediário . . . . . . . . . . . . . . . 28

4 O TEOREMA DE ROLLE $\quad 34$ 
4.1 O Teorema de Rolle . . . . . . . . . . . . . . . . . . . . 34

5 UMA APLICAÇÃO PARTICULAR DO TEOREMA DE ROLLE 37

5.1 Uma aplicação Particular do Teorema de Rolle . . . . . . . . . . . . . . . . 37

6 O TEOREMA DO VALOR MÉDIO 42

6.1 O Teorema do Valor Médio . . . . . . . . . . . . . . . . . . . . . . . 42

7 UMA APLICAÇÃO PARTICULAR DO TEOREMA DO VALOR MÉDIO 48

7.1 Uma Aplicação Particular do Teorema do Valor Médio . . . . . . . . . . . 48

8 APLICAÇÃO DO TEOREMA 8.1.1 NO ENSINO MÉDIO 54

8.1 Aplicação do Teorema 8.1 .1 no Ensino Médio . . . . . . . . . . . . . . . . . 54

9 CONSIDERAÇÕES FINAIS $\quad 60$

REFERÊNCIAS $\quad 61$ 


\section{INTRODUÇÃO}

Muitas situações relacionadas às mais diversas áreas, principalmente, às ciências, podem ser traduzidas, matematicamente, por funções reais contínuas e deriváveis em $\mathbb{R}$ ou em um subconjunto de $\mathbb{R}$, estando sujeitas à aplicação de importantes teoremas do Cálculo, como o Teorema do Valor Extremo, o Teorema do Valor Intermediário, o Teorema de Rolle e o Teorema do Valor Médio, por exemplo, para a obtenção de dados relevantes relativos à essas situações. São exemplos de situações como essas o cálculo da taxa de crescimento populacional de determinado local ou região, em Geografia; o decaimento radioativo de elementos químicos, na Química; a taxa de crescimento populacional de uma cultura de bactérias, na Biologia; a determinação da velocidade de um móvel em um dado instante; na taxa de propagação de uma doença, na Medicina; etc.

O Teorema do Valor Médio é um dos mais importantes resultados do Cálculo Diferencial e é usado, principalmente, como ferramenta para a demonstração de outros teoremas e para comprovar afirmações acerca de funções contínuas em um dado intervalo, utilizando-se de hipóteses locais sobre derivadas em pontos deste intervalo. Um bom exemplo de sua utilização é visto na demonstração da relação existente entre o crescimento e o decrescimento de uma função e o sinal de sua derivada. Ele enuncia uma importante propriedade relativa às funções contínuas, assegurando que, em uma função contínua em um intervalo $[a, b]$ e derivável em $(a, b)$, tomando-se dois pontos $A$ e $B$ quaisquer, existe um ponto de seu gráfico, entre $A$ e $B$ onde a reta tangente ao gráfico possui a mesma inclinação da reta secante $\overleftrightarrow{A B}$

O Teorema de Rolle, assim como o Teorema do valor Médio, é mais um resultado do Cálculo que também auxilia na demonstração de outros Teoremas e pode ser utilizado para comprovação de algumas propriedades relativas às funções contínuas e deriváveis em um determinado intervalo. Este teorema assegura que, para uma função $f$ contínua no intervalo $[a, b]$, derivável no intervalo $(a, b)$ e tendo $f(a)=f(b)$, tomando-se dois pontos $A$ e $B$ quaisquer, existe um ponto do gráfico de $f$ entre $A$ e $B$ onde a inclinação da reta tangente ao gráfico é nula. Restringindo-se o Teorema de Rolle a um caso particular e estendendo o resultado obtido a partir dessa restrição ao Teorema do Valor Médio, chegamos ao resultado principal deste trabalho, que consiste em uma aplicação 
particular do Teorema do Valor Médio.

Como o resultado que será apresentado é aplicado às funções reais, contínuas e deriváveis em um dado intervalo, necessitamos antes da compreensão de alguns conceitos e propriedades relativos aos números e funções reais, limites de funções, continuidade, derivadas, como por exemplo, intervalos, continuidade e tangentes, para dar fundamentação necessária à sua compreensão.

Ainda para estruturação do resultado principal, faz-se necessário explicitar outros teoremas do Cálculo que servem de ferramentas auxiliares para sua construção e/ou demonstração.

Observando-se que o teorema que é o resultado principal deste trabalho aborda conceitos característicos da geometria analítica, apresentaremos ainda, neste trabalho, algumas sugestões de problemas que podem ser aplicados no Ensino Médio, nos quais se utiliza o teorema apresentado em sua solução. 


\section{FUNDAMENTAÇÃO TEÓRICA}

Neste capítulo serão abordados os conceitos e propriedades relativos a números e funções reais, limites de funções, continuidade e derivadas, que são fundamentais para a compreensão do resultado principal desta dissertação.

\subsection{Números Reais}

Vejamos, inicialmente, alguns conceitos e propriedades dos números reais.

\subsubsection{Supremo e ínfimo}

Antes de definirmos supremo e ínfimo faz-se necessário enunciar outros dois conceitos fundamentais para a sua compreensão.

Definição 2.1. (Cota superior): Seja $A \subseteq \mathbb{R}$ não vazio. Um elemento $u \in \mathbb{R}$ é dito cota superior de $A$ se $a \leq u$, para todo $a \in A$. Se um conjunto não vazio possui uma cota superior, pode-se verificar que, na verdade, existe uma infinidade de cotas superiores.

Exemplo: 2.1. No subconjunto $M:=\{x \in \mathbb{R} ; 0<x<4\}$, 4 é uma cota superior.

Definição 2.2. (Cota inferior): Seja $A \subseteq \mathbb{R}$ não vazio. Um elemento $t \in \mathbb{R}$ é dito cota inferior de $A$ se $a \geq t$, para todo $a \in A$. Analogamente ao item anterior, se um conjunto não vazio possui uma cota inferior, existe uma infinidade de cotas inferiores.

Observação: 2.1.1. Nem todo subconjunto de $\mathbb{R}$ possui cota superior ou cota inferior. De fato, o subconjunto $\{x \in \mathbb{R} ; x>3\}$ não possui cota superior.

Definição 2.3. Um subconjunto dos números reais é dito limitado inferiormente se possui cota inferior. Se possui cota superior este conjunto é dito limitado superiormente. Se possui cota superior e inferior este conjunto é dito, apenas, limitado.

Agora vejamos as definções de supremo e ínfimo:

Definição 2.4 (Supremo). Seja $A$ um subconjunto não vazio de $\mathbb{R}$ limitado superiormente. Dado $u \in \mathbb{R}$, dizemos que $u$ é o supremo de $A$, e denota-se por $u=\sup (A)$, se: 
i. $a \leq u$, para todo $a \in A$, isto é, $u$ é uma cota superior;

ii. $a \leq v$, para todo $a \in A$, então $u \leq v$, isto é, $u$ é a menor das cotas superiores.

Definição 2.5 (Ínfimo). Seja $A$ um subconjunto não vazio de $\mathbb{R}$ limitado inferiormente. Dado $t \in \mathbb{R}$, dizemos que $t$ é o ínfimo de $A$, e denota-se por $t=\inf (A)$, se:

i. $a \geq t$, para todo $a \in A$, isto é, $t$ é uma cota inferior;

ii. $a \geq s$, para todo $a \in A$, então $t \geq s$, isto é, $t$ é a maior das cotas inferiores.

Exemplo: 2.2. Considere os conjuntos $R=\{x \in \mathbb{R} ; 2<x<5\}$ e $S=\{x \in \mathbb{R} ; 2 \leq x \leq 5\}$. Vamos verificar que $R$ e $S$ têm o mesmo supremo que é igual a 5. De fato, ambos são limitados superiormente e o 5 é a menor das suas cotas superiores pois, para todo $a \in R$ teremos $a \leq 5$, para todo $b \in S$ teremos $b \leq 5$ e ainda, supondo-se que exista um número real $v>5$ tal que $v$ é a menor das cotas superiores de $R$ e também de $S$, tem-se que $\frac{5+v}{2}$ também é uma cota superior tanto de $R$ quanto de $S$ e $\frac{5+v}{2}<v$, contrariando a hipótese de $v$ ser a menor de suas cotas superiores. Analogamente, verificamos que $R$ e $S$ também possuem o mesmo ínfimo que é igual a 2, visto que ambos são limitados inferiormente, tendo o 2 como a maior das suas cotas inferiores pois, para todo $a \in R$ teremos $a \geq 2$, para todo $b \in S$ teremos $b \geq 2$ e, admitindo-se que exista $s \in \mathbb{R}$, com $s$ a maior das cotas inferiores de $R$ e também de $S$, tem-se que $\frac{s+2}{2}$ é uma cota inferior de ambos os conjuntos $R$ e $S$ e $\frac{s+2}{2}>s$, o que contradiz a hipótese de $s$ ser a maior de suas cotas inferiores.

Observação: 2.1.2. Quando se diz que um subconjunto não vazio de $\mathbb{R}$ tem supremo e/ou infimo, nada se pode afirmar sobre eles pertencerem ou não a este subconjunto.

Agora provaremos que o supremo e o ínfimo, caso existam, são únicos.

Proposição 2.1. (Unicidade do Supremo): Considere A um subconjunto não vazio de R. Se A possui supremo então ele é único.

Demonstração. Seja $u=\sup (A)$ e suponha que $v=\sup (A)$. Como $u$ é uma cota superior de $A$ e $v=\sup (A)$ devemos ter $v \leq u$. Por outro lado, como $v$ é uma cota superior de $A$ e $u=\sup (A)$, também temos $u \leq v$. Essas duas desigualdades implicam que $u=v$ e portanto, o supremo é único (quando existe).

Observação: 2.1.3. A prova da unicidade do infimo é análoga à da unicidade do supremo. 


\subsubsection{Intervalos}

Antes de definirmos intervalo é necessário desenvolver uma noção mais precisa do significado de estar entre dois números reais. Entenda-se que, dados dois números reais distintos $a$ e $b$ quaisquer, com $a<b$, sem perda de generalidade, o conjunto dos números reais que estão entre os números $a$ e $b$ é formado por todos os números reais maiores que ou iguais a $a$ e, ao mesmo tempo, menores que ou iguais a $b$.

Vejamos agora a definição de intervalo.

Definição 2.6. Dizemos que $I \subseteq \mathbb{R}$ é um intervalo se, dados $a, b \in I$ e $c \in \mathbb{R}$ tais que $a \leq c \leq b$, então $c \in I$.

Teorema 2.1.1. Seja I um subconjunto dos números reais com pelo menos dois pontos. Então, I é um intervalo se, e somente se, tem uma, e apenas uma, das seguintes formas:
(i) $(a, b)=\{x \mid a<x<b\}$
(vi) $[a,+\infty)=\{x \mid x \geq a\}$
(ii) $[a, b]=\{x \mid a \leq x \leq b\}$
(vii) $(-\infty, b)=\{x \mid x<b\}$
iii) $(a, b]=\{x \mid a<x \leq b\}$
(viii) $(-\infty, b]=\{x \mid x \leq b\}$
(iv) $[a, b)=\{x \mid a \leq x<b\}$
$(i x)(-\infty,+\infty)=\mathbb{R}$
(v) $(a,+\infty)=\{x \mid x>a\}$

Demonstração. Dividiremos a prova em dois casos:

Caso 1 (Intervalos limitados): Suponha inicialmente que $I$ é um intervalo limitado e seja $a:=\inf (I)$ e $b:=\sup (I)$. Dado $x \in I$ temos que $a \leq x \leq b$ e portanto, $x \in[a, b]$. Como $x$ é arbitrário em $I$, isto prova que $I \subseteq[a, b]$. Vamos provar agora que $(a, b) \subseteq I$. Suponha que exista $y \in(a, b)$ tal que $y \notin I$. Então, por definição de ínfimo e supremo, $y \leq a$ ou $y \geq b$ e portanto, $y \notin(a, b)$, uma contradição. Dessa forma concluímos que $(a, b) \subseteq I \subseteq[a, b]$. Para finalizar a prova, consideremos os casos a seguir:

(I) se $a \notin I$ e $b \notin I$ então $I=(a, b)$.

(II) se $a \in I$ e $b \in I$ então $I=[a, b]$.

(III) se $a \notin I$ e $b \in I$ então $I=(a, b]$.

(IV) se $a \in I$ e $b \notin I$ então $I=[a, b)$. 
Caso 2 (Intervalos ilimitados): Suponha agora que $I$ é um intervalo ilimitado e considere as circunstâncias seguintes:

1) Seja $I$ um intervalo limitado inferiormente e seja $a:=\inf (I)$. Dado $x \in I$ temos que $a \leq x$ e portanto, $x \in[a,+\infty)$. Como $x$ é arbitrário em $I$, isto prova que $I \subseteq[a,+\infty)$. Vamos provar agora que $(a,+\infty) \subseteq I$. Suponha que exista $y \in(a,+\infty)$ tal que $y \notin I$. Então, por definição de ínfimo, $y \leq a$ e portanto, $y \notin(a,+\infty)$, uma contradição. Dessa forma concluímos que $(a,+\infty) \subseteq I \subseteq[a,+\infty)$. Para finalizar a prova, consideremos os casos a seguir:

(I) se $a \notin I$ então $I=(a,+\infty)$.

(II) se $a \in I$ então $I=[a,+\infty)$.

2) Seja $I$ um intervalo limitado superiormente e seja $b:=\sup (I)$. Dado $x \in I$ temos que $x \leq b$ e portanto, $x \in(-\infty, b]$. Como $x$ é arbitrário em $I$, isto prova que $I \subseteq(-\infty, b]$. Vamos provar agora que $(-\infty, b) \subseteq I$. Suponha que exista $y \in(-\infty, b)$ tal que $y \notin I$. Então, por definição de supremo, $y \geq b$ e portanto, $y \notin(-\infty, b)$, uma contradição. Dessa forma concluímos que $(-\infty, b) \subseteq I \subseteq(-\infty, b]$. Para finalizar a prova, consideremos os casos a seguir:

(I) se $b \notin I$ então $I=(-\infty, b)$.

(II) se $b \in I$ então $I=(-\infty, b]$.

3) Seja I um intervalo que não possui limite inferior nem limite superior. Vamos demonstrar que $I=\mathbb{R}$, e para isto é suficiente verificar que $\mathbb{R} \subseteq I$ uma vez que $I \subseteq \mathbb{R}$. Seja $y$ um número real arbitrário. Por hipótese existem $p, q \in I$ tais que $p \leq y \leq q$. Como $I$ é um intervalo devemos ter $y \in I$. Portanto, $\mathbb{R} \subseteq I$ como esperávamos demonstrar.

\section{Observações: 2.1.1.}

i) $-\infty$ e $+\infty$ são apenas símbolos, e não números.

ii) Os intervalos $(a, b),[a, b],(a, b]$ e $[a, b)$ são limitados, com a e b como extremos e os intervalos $(-\infty, b),(-\infty, b],(a,+\infty),[a,+\infty)$ e $(-\infty,+\infty)$ são ilimitados. 
iii) O intervalo $(-\infty, a)$ é a semi-reta esquerda, aberta, de origem a. Os demais intervalos ilimitados têm denominações análogas.

iv) Os intervalos $(a, b),(-\infty, b),(a,+\infty) e(-\infty,+\infty)$ são chamados de intervalos abertos, os intervalos $(a, b],[a, b),(-\infty, b] e[a,+\infty)$ são chamados de intervalos semiabertos e o intervalo $[a, b]$ é chamado de intervalo fechado.

v) Quando no intervalo fechado $[a, b]$ tivermos $a=b$, esse intervalo se reduzirá a um único elemento, e será chamado de intervalo degenerado e os outros três intervalos limitados serão vazios.

\section{Exemplo: 2.3.}

- $(-2,1)$

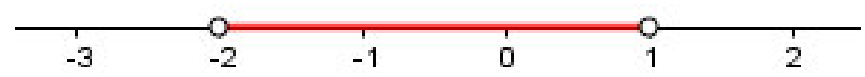

Figura 2.1: Intervalo $(-2,1)$

- $[-2,1]$

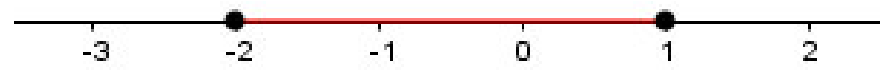

Figura 2.2: Intervalo $[-2,1]$

- $(-2,1]$

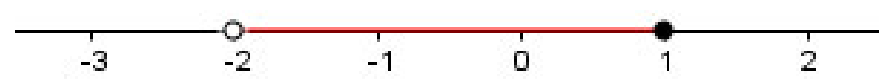

Figura 2.3: Intervalo $(-2,1]$

- $[-2,1)$

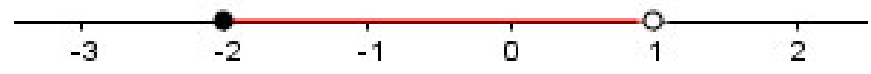

Figura 2.4: Intervalo $[-2,1)$

- $(2,+\infty)$

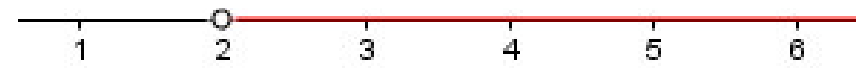

Figura 2.5: Intervalo $(2,+\infty)$ 
- $[2,+\infty)$

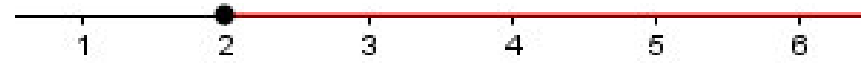

Figura 2.6: Intervalo $[2,+\infty)$

- $(-\infty, 2)$

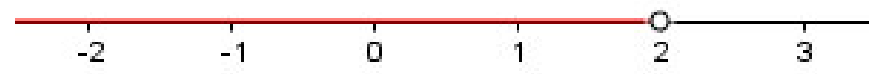

Figura 2.7: Intervalo $(-\infty, 2)$

- $(-\infty, 2]$

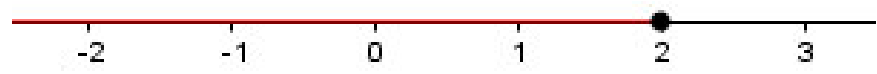

Figura 2.8: Intervalo $(-\infty, 2]$

\subsubsection{Desigualdade triangular}

O módulo ou o valor absoluto de um número real $x$, qualquer, está associado ao conceito de distância desse número até a origem do sistema e é denotado por $|x|$. Ciente de que a distância é uma medida não negativa, o módulo de um número é sempre maior ou igual a zero, sendo que é igual a zero somente no caso desse número ser o próprio zero. Temos que:

$$
|x|=\left\{\begin{array}{c}
x, \text { se } x \geq 0 \\
-x, \text { se } x<0
\end{array},\right.
$$

Proposição 2.2. Para todo $x \in \mathbb{R}$ temos $-|x| \leq x \leq|x|$.

Demonstração. Com efeito, se $x>0$ então $|x|=x$ e $-|x|=-x$. Logo, $-|x|<x=|x|$. Agora, se $x<0$ então $|x|=-x$ e $-|x|=x$. Logo, $-|x|=x<|x|$.

Com relação ao módulo de um número real, um resultado importante para este trabalho é a chamada desigualdade triangular. 
Proposição 2.3. (Desigualdade triangular) Dados $a, b \in \mathbb{R}$, temos que:

$$
|a+b| \leq|a|+|b|
$$

Demonstração. Da proposição 2.2 temos que $-|a| \leq a \leq|a|$ e $-|b| \leq b \leq|b|$. Daí, segue que, se $-|a|-|b| \leq a+b \leq|a|+|b|$ então $-(|a|+|b|) \leq a+b \leq|a|+|b|$, ou ainda, $|a+b| \leq|a|+|b|$.

\subsection{Limites de Funções}

Antes de definirmos o limite de uma função veremos alguns conceitos essenciais para sua compreensão.

Definição 2.7. Dado um número real $\epsilon>0$, chama-se $\epsilon$-vizinhança de $a \in \mathbb{R}$ o intervalo $(a-\epsilon, a+\epsilon)$, denotado por $V_{\epsilon}(a)$.

Observemos que a condição $x \in V_{\epsilon}(a)$ pode ser escrita das três formas seguintes:

$$
|x-a|<\epsilon ; \quad-\epsilon<x-a<\epsilon ; \quad a-\epsilon<x<a+\epsilon
$$

Observação: 2.2.1. Às vezes interessa considerar uma $\epsilon$-vizinhança de a, excluindo-se o própio ponto a, que se chama vizinhança furada, denotada por

$$
V_{\epsilon}^{\prime}(a)=V_{\epsilon}(a)-\{a\}=\{x: 0<|x-a|<\epsilon\}
$$

Definição 2.8. Seja $A$ um subconjunto não vazio de $\mathbb{R}$. Dizemos que um número $a$ qualquer é ponto de acumulação de $A$ se, dado $\epsilon>0, V_{\epsilon}^{\prime}(a)$ contém algum elemento de $A$.

Observação: 2.2.2. Indica-se com $A^{\prime}$ o conjunto dos pontos de acumulação de A. Portanto, se $a \in A^{\prime}$ então $a \in A-\{a\}$.

Exemplo: 2.4. Seja $C=(0,2)$. Tem-se que 2 é ponto de acumulação de $C$ pois, dado $\epsilon>0$ arbitrário, $V_{\epsilon}^{\prime}(2)$ contém algum elemento de $C$. 
Definição 2.9. Sejam $A$ um subconjunto não-vazio de $\mathbb{R}$, uma função $f: A \rightarrow \mathbb{R}$ e $a$ um ponto de acumulação de $A$ (que pode ou não pertencer a $A$ ). Dizemos que um número $L$ é o limite de $f(x)$ quando $x$ tende a $a$ se, dado $\epsilon>0$ arbitrário, existe $\delta>0$ tal que, se $x \in A \cap V_{\delta}^{\prime}(a)$ então $|f(x)-L|<\epsilon$.

Denotamos por $\lim _{x \rightarrow a} f(x)=L$ (lê-se: limite de $f(x) \operatorname{com} x$ tendendo a $a$ é igual a $L$ ).

Exemplo: 2.5. Mostre que $\lim _{x \rightarrow 1}(4 x-3)=1$, usando a definição 2.5.

Solução: 2.2.1. Dado $\epsilon>0$ arbitrário considere $\delta=\frac{\epsilon}{4}$. Se $0<|x-1|<\delta$ então $|(4 x-3)-1|=|4 x-4|=4|x-1|<4 \delta=\epsilon$, e portanto, $\lim _{x \rightarrow 1}(4 x-3)=1$.

\subsubsection{Propriedades dos limites}

Apresentaremos as propriedades dos limites necessárias à compreensão deste trabalho através da proposição a seguir.

Proposição 2.4. Sejam A um subconjunto não vazio de $\mathbb{R}, f, g: A \rightarrow \mathbb{R}, a \in A^{\prime}$, com $\lim _{x \rightarrow a} f(x)=L_{1}$ e $\lim _{x \rightarrow a} g(x)=L_{2}$ então:

i. $\lim _{x \rightarrow a}(f+g)(x)=L_{1}+L_{2}$

ii. $\lim _{x \rightarrow a} c f(x)=c \cdot L_{1}$, para todo $c \in \mathbb{R}$;

iii. $\lim _{x \rightarrow a}(f \cdot g)(x)=L_{1} \cdot L_{2}$;

iv. $\lim _{x \rightarrow a} c=c$, para todo $c \in \mathbb{R}$.

Demonstração.

(i) Seja $\epsilon>0$ um número real arbitrário. Por hipótese, existe $\delta_{1}>0$ tal que $\left|f(x)-L_{1}\right|<\frac{\epsilon}{2}$ sempre que $x \in A \cap V_{\delta_{1}}^{\prime}(a)$. Da mesma forma, existe $\delta_{2}>0$ tal que $\left|g(x)-L_{2}\right|<\frac{\epsilon}{2}$ sempre que $x \in A \cap V_{\delta_{2}}^{\prime}(a)$. Seja $\delta=\min \left\{\delta_{1}, \delta_{2}\right\}$. Se $x \in A \cap V_{\delta}^{\prime}(a)$, então

$$
\left|(f+g)(x)-\left(L_{1}+L_{2}\right)\right|=\left|\left(f(x)-L_{1}\right)+\left(g(x)-L_{2}\right)\right| \leq\left|f(x)-L_{1}\right|+\left|g(x)-L_{2}\right|<\epsilon .
$$

Como $\epsilon$ é arbitrário segue-se que

$$
\lim _{x \rightarrow a}(f+g)(x)=L_{1}+L_{2}=\lim _{x \rightarrow a} f(x)+\lim _{x \rightarrow a} g(x) .
$$


(ii) Dividiremos a prova em dois casos:

Caso 1 $(c=0)$ : Se $c=0$ então, dado $\epsilon>0$ arbitrário, para todo $\delta>0$ teremos $\left|0 \cdot f(x)-0 \cdot L_{1}\right|=0<\epsilon$ sempre que $x \in A \cap V_{\delta}^{\prime}(a)$.

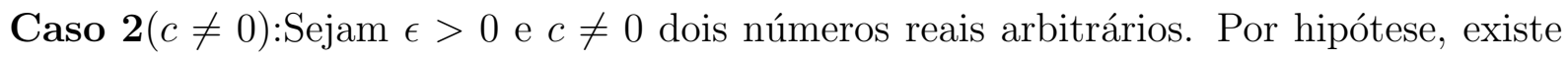
$\delta>0$ tal que $\left|f(x)-L_{1}\right|<\frac{\epsilon}{|c|}$ sempre que $x \in A \cap V_{\delta}^{\prime}(a)$. Se $x \in A \cap V_{\delta}^{\prime}(a)$ então

$$
\left|(c f)(x)-c L_{1}\right|=|c| \cdot\left|f(x)-L_{1}\right| \leq|c| \cdot \epsilon /|c|=\epsilon
$$

. Como $\epsilon$ é arbitrário segue-se que $\lim _{x \rightarrow a} c f(x)=c \cdot L_{1}$.

(iii) Seja um número real arbitrário $\epsilon=p+k, p>0$ e $k>0$, então $\epsilon>0$. Por hipótese, existem $\delta_{1}, \delta_{2}, \delta_{3}>0$ tais que, se $x \in A \cap V_{\delta_{1}}^{\prime}(a)$ então $\left|f(x)-L_{1}\right|<1$, se $x \in A \cap V_{\delta_{2}}^{\prime}(a)$ então $\left|g(x)-L_{2}\right|<\frac{p}{\left|L_{1}\right|+1}$ e se $x \in A \cap V_{\delta_{3}}^{\prime}(a)$ então $\left|f(x)-L_{1}\right|<\frac{k}{\left|L_{2}\right|+1}$. Como $\left|f(x)-L_{1}\right|<1$ temos que $-1<f(x)-L_{1}<1$, ou ainda $-1+L_{1}<f(x)<1+L_{1}$. Daí, teremos $f(x)<1+L_{1}$ e, desta forma, $|f(x)|<\left|1+L_{1}\right| \leq 1+\left|L_{1}\right|$.

Seja $\delta=\min \left\{\delta_{1}, \delta_{2}, \delta_{3}\right\}$ e observando-se que $\left|L_{2}\right|<\left|L_{2}\right|+1$ e $\frac{f(x)}{\left(\left|L_{1}\right|+1\right)}<1$, se $x \in A \cap V_{\delta}^{\prime}$ então,

$$
\begin{aligned}
\left|f(x) \cdot g(x)-L_{1} \cdot L_{2}\right| & =\left|f(x) \cdot\left(g(x)-L_{2}\right)+L_{2} \cdot\left(f(x)-L_{1}\right)\right| \\
& \leq|f(x)| \cdot\left|g(x)-L_{2}\right|+\left(\left|L_{2}\right|+1\right) \cdot\left|f(x)-L_{1}\right| \\
& <|f(x)| \cdot \frac{p}{\left|L_{1}\right|+1}+\left(\left|L_{2}\right|+1\right) \cdot \frac{k}{\left(\left|L_{2}\right|+1\right)} \\
& <p+k .
\end{aligned}
$$

e, portanto, $\left|f(x)(x)-L_{1} \cdot L_{2}\right|<\epsilon$.

Como $\epsilon$ é arbitrário segue-se que $\lim _{x \rightarrow a}(f \cdot g)(x)=L_{1} \cdot L_{2}$.

(iv) Dado $\epsilon>0$ arbitrário, para todo $\delta>0$ teremos $|c-c|=0<\epsilon$ sempre que $x \in A \cap V_{\delta}^{\prime}$. Como $\epsilon$ é arbitrário segue-se que $\lim _{x \rightarrow a} c=c$. 


\subsection{Continuidade}

Para definirmos $\lim _{x \rightarrow a} f(x)$ analisamos o comportamento da função para valores de $x$ próximos de $a$, mas diferentes de $a$. Sabemos que $\lim _{x \rightarrow a} f(x)$ pode existir, mesmo que a função $f$ não seja definida no ponto $a$. Uma outra situação possível é que, se $f$ está definida no ponto $a$ e existe $\lim _{x \rightarrow a} f(x)$, pode acontecer deste limite ser diferente de $f(a)$. Agora, se a função $f$ estiver definida no ponto $a$, existe $\lim _{x \rightarrow a} f(x)$ e este limite for igual a $f(a)$, diremos que a função $f$ é contínua no ponto $a$. Mais precisamente:

Definição 2.10. Seja $A$ um subconjunto não vazio de $\mathbb{R}$. Dizemos que $f: A \rightarrow \mathbb{R}$ é contínua em $a \in A$ se $\lim _{x \rightarrow a} f(x)$ existe e é igual a $f(a)$.

Observação: 2.3.1. Dizemos que $f$ é descontínua em a se $f$ não é contínua em a.

Vejamos, a seguir, alguns exemplos de funções descontínuas.

Exemplo: 2.6. A função $f(x)=\frac{x^{2}-4}{x-2}$ é descontínua no ponto 2, uma vez que não está definida neste ponto.

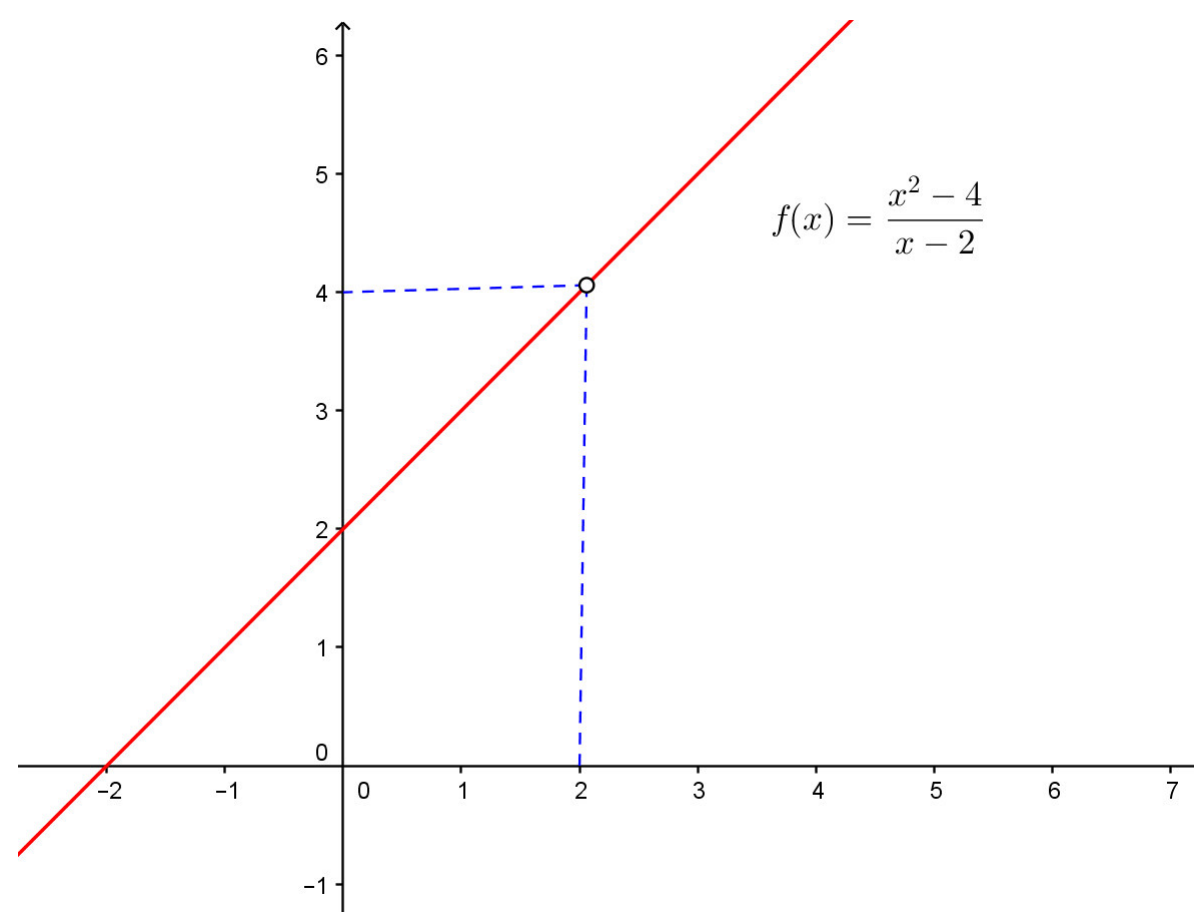

Figura 2.9: Função descontínua em 2 
Exemplo: 2.7. A função $f: \mathbb{R}-\{0\} \rightarrow \mathbb{R}-\{0\}$ definida por $f(x)=\frac{1}{x}$ é descontínua no ponto 0 , pois $\lim _{x \rightarrow 0^{-}} f(x) \neq \lim _{x \rightarrow 0^{+}} f(x)$, logo não existe $\lim _{x \rightarrow 0} f(x)$.

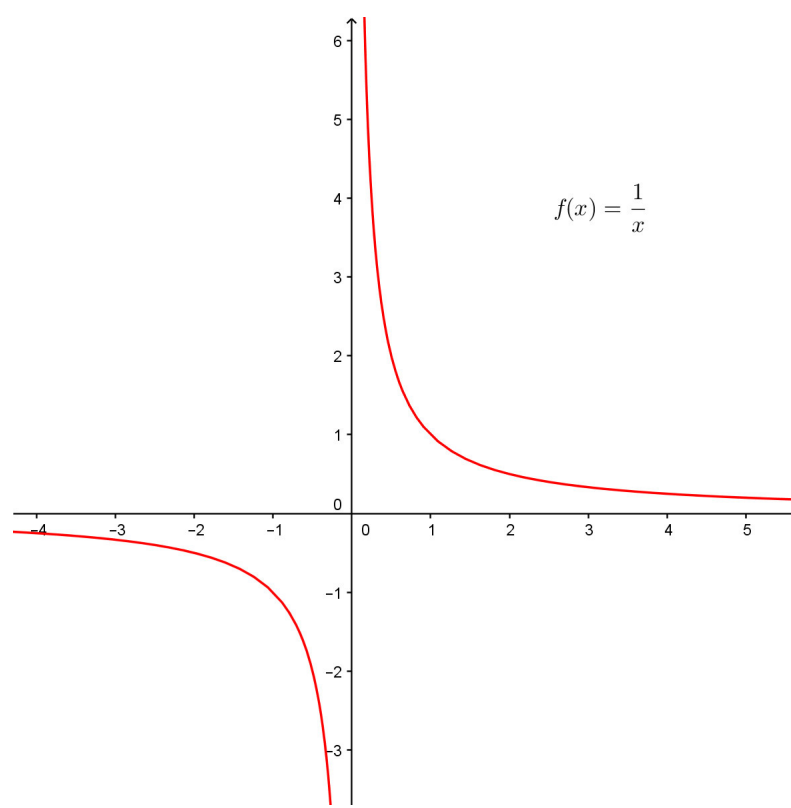

Figura 2.10: Função descontínua em 0

Exemplo: 2.8. A função $f$, representada graficamente na figura a seguir, é descontínua no ponto 1 , pois $\lim _{x \rightarrow 1} f(x)=2 \neq 1=f(1)$.

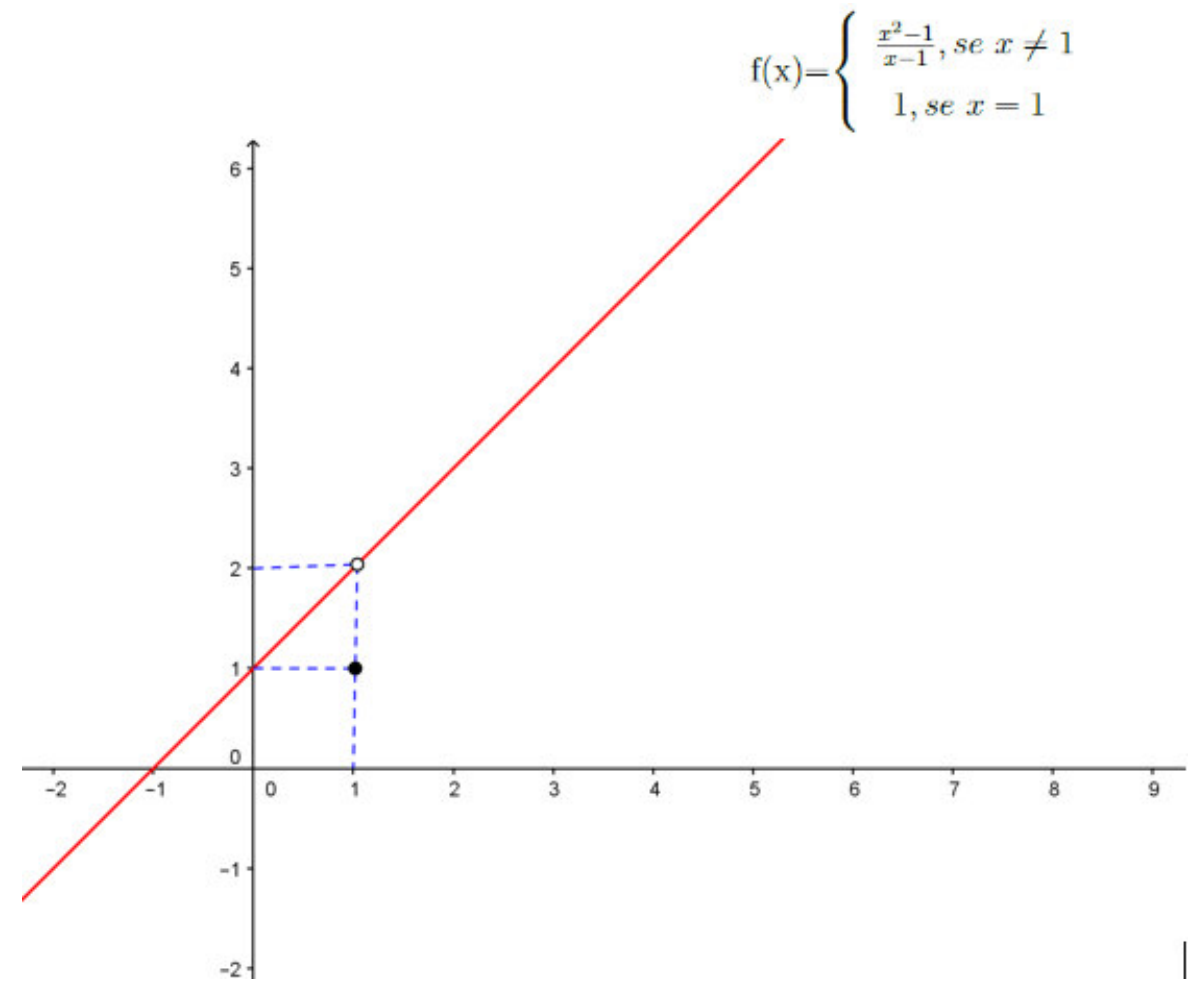

Figura 2.11: Função descontínua em 1 
Proposição 2.5. Sejam $A$ um subconjunto não vazio de $\mathbb{R}$ e $f, g: A \rightarrow \mathbb{R}$ funções contínuas em um ponto $a \in A$. Então

i. $f \pm g$ é contínua em a;

ii. cf é contínua em a;

iii. $f \cdot g$ é contínua em a;

iv. $f / g, \operatorname{com} g(a) \neq 0$, é contínua em $a$.

Observação: 2.3.2. A demonstração desta proposição é uma consequência direta da definição 2.10 e da proposição 2.4.

\subsection{Derivada}

Antes de definirmos a derivada de uma função, vejamos a definição de tangente.

\subsubsection{Tangentes}

Para determinarmos a tangente a uma curva $C$ de equação $y=f(x)$ em um ponto $A(a, f(a))$, tomamos um ponto $B \in C, B(x, f(x))$, com $x \neq a$, e calculamos o coeficiente angular da reta $A B$, ou seja,

$$
m_{A B}=\frac{f(x)-f(a)}{x-a}
$$

Então, aproximamos o ponto $B$ do ponto $A$, ao longo de $C$, fazendo $x$ tender a $a$. Se $m_{A B}$ tender a um número $m$, então podemos definir a tangente $t$ à curva $C$ como a reta de coeficiente angular $m$ que passa pelo ponto $A$, o que implica em dizer que a reta tangente é a posição-limite da reta secante $A B$ quando $B$ tende a $A$.

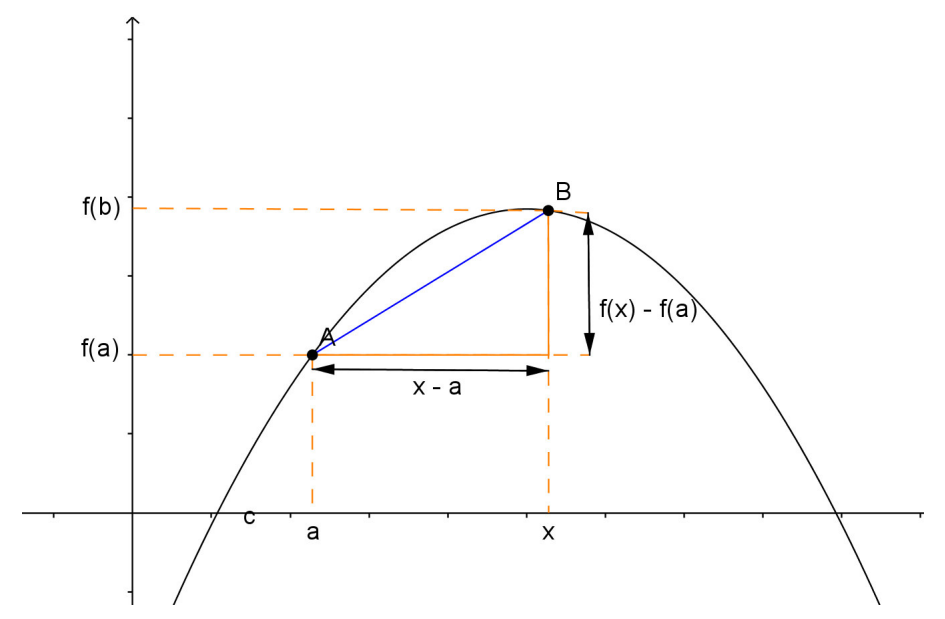

Figura 2.12: Reta tangente à curva $\mathrm{y}=\mathrm{f}(\mathrm{x})$ em um ponto a 


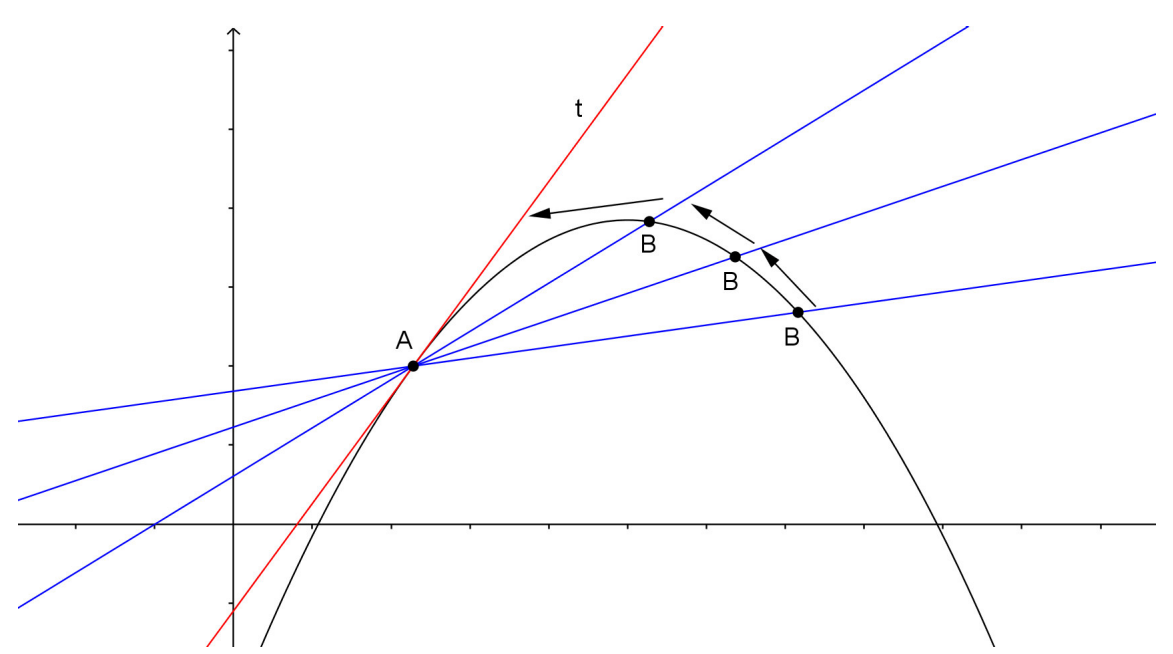

Figura 2.13: Reta tangente à curva $\mathrm{y}=\mathrm{f}(\mathrm{x})$ em um ponto a

Definição 2.11. Dada uma curva de equação $y=f(x)$. A reta tangente à esta curva em um ponto $A(a, f(a))$ tem coeficiente angular igual a

$$
m=\lim _{x \rightarrow a} \frac{f(x)-f(a)}{x-a}
$$

caso esse limite exista.

Fazendo $h=x-a$, temos $x=a+h$ teremos

$$
m=\lim _{h \rightarrow 0} \frac{f(a+h)-f(a)}{h}
$$

\subsubsection{Equação da reta tangente}

Sejam $A$ um subconjunto não vazio de $\mathbb{R}$ e uma função $f: A \rightarrow \mathbb{R}$ contínua em $a \in A$. A reta tangente à curva $y=f(x)$ no ponto $P(a, f(a))$ será:

A reta que passa pelo ponto $P$ e tem coeficiente angular igual a $m(a)=\lim _{h \rightarrow 0} \frac{f(a+h)-f(a)}{h}$, caso esse limite exista. Assim, temos a equação

$$
y-f(a)=m(x-a) .
$$

A reta vertical $x=a$, se $\lim _{h \rightarrow 0} \frac{f(a+h)-f(a)}{h}$ for infinito.

\subsubsection{A derivada de uma função em um ponto}

Sejam $A$ um subconjunto não vazio de $\mathbb{R}$ e uma função $f: A \rightarrow \mathbb{R}$ contínua em $a \in A$. A derivada de $f$ no ponto $a$ é denotada por $f^{\prime}(a)$ (lê-se $f$ linha de $a$ ), e seu valor é 


$$
f^{\prime}(a)=\lim _{h \rightarrow 0} \frac{f(a+h)-f(a)}{h}
$$

caso este limite exista.

Fazendo $x=a+h$, temos $h=x-a$ e, desta forma, $h \rightarrow 0$ se, e somente se, $x \rightarrow a$. Então, uma forma equivalente de denotar a derivada de uma função em um ponto $a$ é

$$
f^{\prime}(a)=\lim _{x \rightarrow a} \frac{f(x)-f(a)}{x-a}
$$

\subsubsection{Derivadas laterais}

Definição 2.12. Sejam $A$ um subconjunto não vazio de $\mathbb{R}$ e uma função $f: A \rightarrow \mathbb{R}$ definida no ponto $a \in A$. Então, a derivada à direita de $f$ em a, denotada por $f_{+}^{\prime}(a)$ é definida da seguinte forma:

$$
f_{+}^{\prime}(a)=\lim _{h \rightarrow 0^{+}} \frac{f(a+h)-f(a)}{h}=\lim _{x \rightarrow a^{+}} \frac{f(x)-f(a)}{x-a}
$$

caso este limite exista.

Definição 2.13. Seja $f$ uma função $y=f(x)$ definida no ponto $a$. Então, a derivada $\grave{a}$ esquerda de $f$ em a, denotada por $f_{-}^{\prime}(a)$ é definida da seguinte forma:

$$
f_{-}^{\prime}(a)=\lim _{h \rightarrow 0^{-}} \frac{f(a+h)-f(a)}{h}=\lim _{x \rightarrow a^{-}} \frac{f(x)-f(a)}{x-a},
$$

caso este limite exista.

Observação: 2.4.1. Se as derivadas laterais de uma função $f$, em um ponto a qualquer, existem e são iguais, dizemos que $f$ é uma função derivável no ponto a. Caso as derivadas laterais de $f$ existam e sejam diferentes, em um ponto a qualquer, dizemos que este ponto a é um ponto anguloso do gráfico da função $f$.

\subsubsection{Continuidade de funções deriváveis}

Teorema 2.4.1. Toda função derivável em um ponto $x_{0}$ é contínua nesse ponto.

Demonstração. Sejam $A$ um subconjunto não vazio de $\mathbb{R}$, uma função $f: A \rightarrow \mathbb{R}$ e um ponto $x_{0} \in A$. Por hipótese, temos que $f$ é derivável em $x_{0}$. Então,

$$
f^{\prime}\left(x_{0}\right)=\lim _{x \rightarrow x_{0}} \frac{f(x)-f\left(x_{0}\right)}{x-x_{0}}
$$

Temos, ainda, que: 


$$
\begin{aligned}
\lim _{x \rightarrow x_{0}}\left[f(x)-f\left(x_{0}\right)\right] & =\lim _{x \rightarrow x_{0}}\left[\left(x-x_{0}\right) \cdot \frac{f(x)-f\left(x_{0}\right)}{x-x_{0}}\right] \\
& =\lim _{x \rightarrow x_{0}}\left(x-x_{0}\right) \cdot \lim _{x \rightarrow x_{0}} \frac{f(x)-f\left(x_{0}\right)}{x-x_{0}} \\
& =0 \cdot f^{\prime}\left(x_{0}\right) .
\end{aligned}
$$

Portanto, $\lim _{x \rightarrow x_{0}}\left[f(x)-f\left(x_{0}\right)\right]=0$.

Desta forma, temos

$$
\begin{aligned}
\lim _{x \rightarrow x_{0}} f(x) & =\lim _{x \rightarrow x_{0}}\left[f(x)-f\left(x_{0}\right)+f\left(x_{0}\right)\right] \\
& =\lim _{x \rightarrow x_{0}}\left[f(x)-f\left(x_{0}\right)\right]+\lim _{x \rightarrow x_{0}} f\left(x_{0}\right) \\
& =f\left(x_{0}\right) .
\end{aligned}
$$

Observação: 2.4.2. A recíproca do teorema 2.4.1 não é verdadeira. Como contraexemplo tomemos a função modular $f(x)=|x|$, que é contínua mas não é derivável em 0 pois, como

$$
f(x)=\left\{\begin{array}{c}
x, \text { se } x \geq 0 \\
-x, \text { se } x<0
\end{array},\right.
$$

então, $f_{+}^{\prime}(0)=x^{\prime}=1$ e $f_{-}^{\prime}(0)=(-x)^{\prime}=-1$. Logo, como $f_{+}^{\prime}(0) \neq f_{-}^{\prime}(0)$, não existe $f^{\prime}(0)$.

\subsubsection{Regras de derivação}

i) Derivada de uma função constante.

Seja $f: \mathbb{R} \rightarrow \mathbb{R}$ uma função constante, ou seja, uma função da forma $f(x)=c$, $c \in \mathbb{R}$. Então, sua derivada é $f^{\prime}(x)=0$.

ii) Derivada de uma função potência.

Seja $f: \mathbb{R} \rightarrow \mathbb{R}$ uma função potência, ou seja, uma função da forma $f(x)=x^{n}$, $n \in \mathbb{N}$. Então, sua derivada é $f^{\prime}(x)=n \cdot x^{n-1}$.

iii) Derivada do produto de uma constante por uma função.

Sejam $A$ um subconjunto não vazio de $\mathbb{R}$, uma constante $c \in \mathbb{R}$ e as funções $f, g: A \rightarrow \mathbb{R}$ com $g$ definida por $g(x)=c f(x)$. Se existe $f^{\prime}(x)$, então a derivada da função $g$ é $g^{\prime}(x)=c f^{\prime}(x)$.

iv) Derivada de uma soma.

Sejam $A$ um subconjunto não vazio de $\mathbb{R}$, as funções $f, g: A \rightarrow \mathbb{R}$ e $h$ uma função definida por $h(x)=f(x)+g(x)$. Se existem $f^{\prime}(x)$ e $g^{\prime}(x)$, então a derivada da função $h$ é $h^{\prime}(x)=f^{\prime}(x)+g^{\prime}(x)$. 
v) Derivada de um produto.

Sejam $A$ um subconjunto não vazio de $\mathbb{R}$, as funções $f, g: A \rightarrow \mathbb{R}$ e $h$ uma função definida por $h(x)=f(x) \cdot g(x)$. Se existem $f^{\prime}(x)$ e $g^{\prime}(x)$, então a derivada da função $h$ é $h^{\prime}(x)=f(x) \cdot g^{\prime}(x)+f^{\prime}(x) \cdot g(x)$.

vi) Derivada da função exponencial.

Sejam $A$ um subconjunto não vazio de $\mathbb{R}$ e uma função $f: A \rightarrow \mathbb{R}$ definida por $f(x)=a^{x}, a>0$ e $a \neq 1$. Então, sua derivada é $f^{\prime}(x)=a^{x} \cdot \ln a, a>0$ e $a \neq 1$. 


\section{CONSEQUÊNCIAS DA CONTINUIDADE}

Veremos, neste capítulo, conceitos e teoremas relativos à continuidade, que também fundamentam o resultado principal deste trabalho.

\subsection{Valores Extremos}

Definiremos a seguir os valores extremos de uma função.

Definição 3.1. Seja $A$ um subconjunto não vazio de $\mathbb{R}$. Dizemos que uma função $f: A \subseteq \mathbb{R} \rightarrow \mathbb{R}$ tem:

(i) máximo absoluto (ou máximo global) em $c$ se $f(c) \geq f(x)$, para todo $x \in A$.

O valor $f(c)$ é chamado valor máximo de $f$ em $A$.

(ii) mínimo absoluto (ou mínimo global) em $c$ se $f(c) \leq f(x)$, para todo $x \in A$.

O valor $f(c)$ é chamado valor mínimo de $f$ em $A$.

Os valores máximo e mínimo de $f$ são chamados valores extremos de $f$.

Definição 3.2. Sejam $A$ um subconjunto não vazio de $\mathbb{R}$ e $I \subset A$ um intervalo aberto que contém um ponto $c$, qualquer. Dizemos que uma função $f: A \subseteq \mathbb{R} \rightarrow \mathbb{R}$ tem:

(i) máximo local em $c$ se $f(c) \geq f(x)$, para todo $x \in I$.

(ii) mínimo local em $c$ se $f(c) \leq f(x)$, para todo $x \in I$.

Exemplo: 3.1. Observe os pontos de máximo e mínimo, globais e locais, no gráfico de $f(x)=3 x^{4}-16 x^{3}+18 x^{2}$, definida em $A=[-1,4]$, conforme a figura 3.1 .

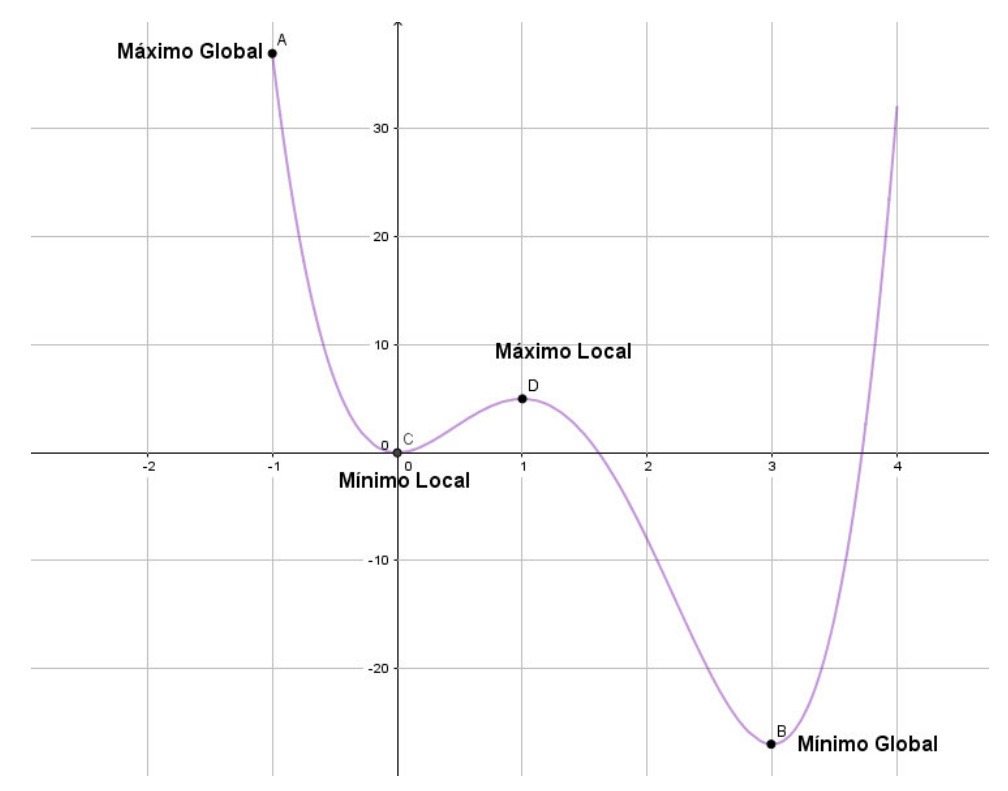

Figura 3.1: Gráfico de $f(x)=3 x^{4}-16 x^{3}+18 x^{2}$, definida em $A=[-1,4]$ 


\subsection{O Teorema do Valor Extremo}

Apresentaremos, agora, o Teorema do Valor Extremo.

Teorema 3.2.1 (Teorema do Valor Extremo). Seja $f:[a, b] \rightarrow \mathbb{R}$ uma função contínua, então $f$ assume um valor máximo absoluto e um valor mínimo absoluto no intervalo $[a, b]$.

Antes de demonstrarmos este teorema, apresentaremos um resultado fundamental para sua demonstração que consiste no teorema a seguir.

Teorema 3.2.2. Se uma função $f:[a, b] \rightarrow \mathbb{R}$ é contínua, então $f$ é limitada.

Demonstração. Suponhamos que $f$ seja ilimitada. Então, existe uma sequência $\left(x_{n}\right)$ tal que $\left|f\left(x_{n}\right)\right|$ tende a $\infty$. Por outro lado, como $\left(x_{n}\right)$ é uma sequência contida no intervalo $[a, b]$, pelo teorema de Bolzano-Weierstrass, existe $\left(n_{j}\right)$ tal que a subsequência $\left(x_{n_{j}}\right)$ é convergente, ou seja, existe $x_{0} \in[a, b]$ tal que $\left(x_{n_{j}}\right)$ converge para $x_{0}$. No entanto, como $f$ é contínua, temos que $\lim _{j \rightarrow \infty}\left|f\left(x_{n_{j}}\right)\right|=\infty=f\left(x_{0}\right)$, o que é um absurdo! Logo, $f$ é limitada.

Agora demonstraremos o Teorema do Valor Extremo.

Demonstração. Como $f$ é contínua, pelo Teorema 3.2.2, temos que $f$ é limitada e, portanto, existem $\sup (i m(f))$ e $\inf (i m(f))$, de modo que

$$
\inf (i m(f)) \leq f(x) \leq \sup (i m(f)),
$$

para todo $x \in[a, b]$.

Seja $K=\sup (\operatorname{Im}(f))$. Suponhamos $f(x) \neq K$ para todo $x \in[a, b]$ e considere uma função $g$, definida por

$$
g(x)=\frac{1}{K-f(x)}
$$

Como $f(x)<K$, para todo $x \in[a, b]$, então $K-f(x)>0$, e assim, temos que $g$ é contínua. Logo, pelo Teorema 3.2.2, $g$ é limitada.

Mas, se $g$ é limitada, então para algum $k$, teremos

$$
0<\frac{1}{K-f(x)}<k
$$

e portanto, $f(x)<K-1 / k<K$ para todo $x \in[a, b]$, contrariando o fato de $K$ ser supremo de $(\operatorname{Im}(f))$. Portanto, deve haver $d \in[a, b]$ tal que $f(d)=K$

Analogamente, seja $W=\inf (i m(f)), \operatorname{com} f(x) \neq W$ para todo $x \in[a, b]$. Tomemos uma função

$$
h(x)=\frac{1}{f(x)-W}
$$

Como $f(x)>W$, para todo $x \in[a, b]$, então $f(x)-W>0$, e assim, temos que $h$ é contínua. Logo, pelo Teorema 3.2.2, $h$ é limitada. 
Por outro lado, se $h$ é limitada, teremos assim, para algum $w$,

$$
0<\frac{1}{f(x)-W)}<w
$$

e portanto, $f(x)>W+1 / w>W$ para todo $x \in[a, b]$, o que contradiz a hipótese de $W$ ser ínfimo de $(\operatorname{Im}(f))$. Portanto, deve haver $c \in[a, b]$ tal que $f(c)=W$.

O Teorema de Bolzano-Weierstrass pode ser visto em [7] (p.36).

O Teorema do Valor Extremo está ilustrado logo abaixo, na figura 3.2.

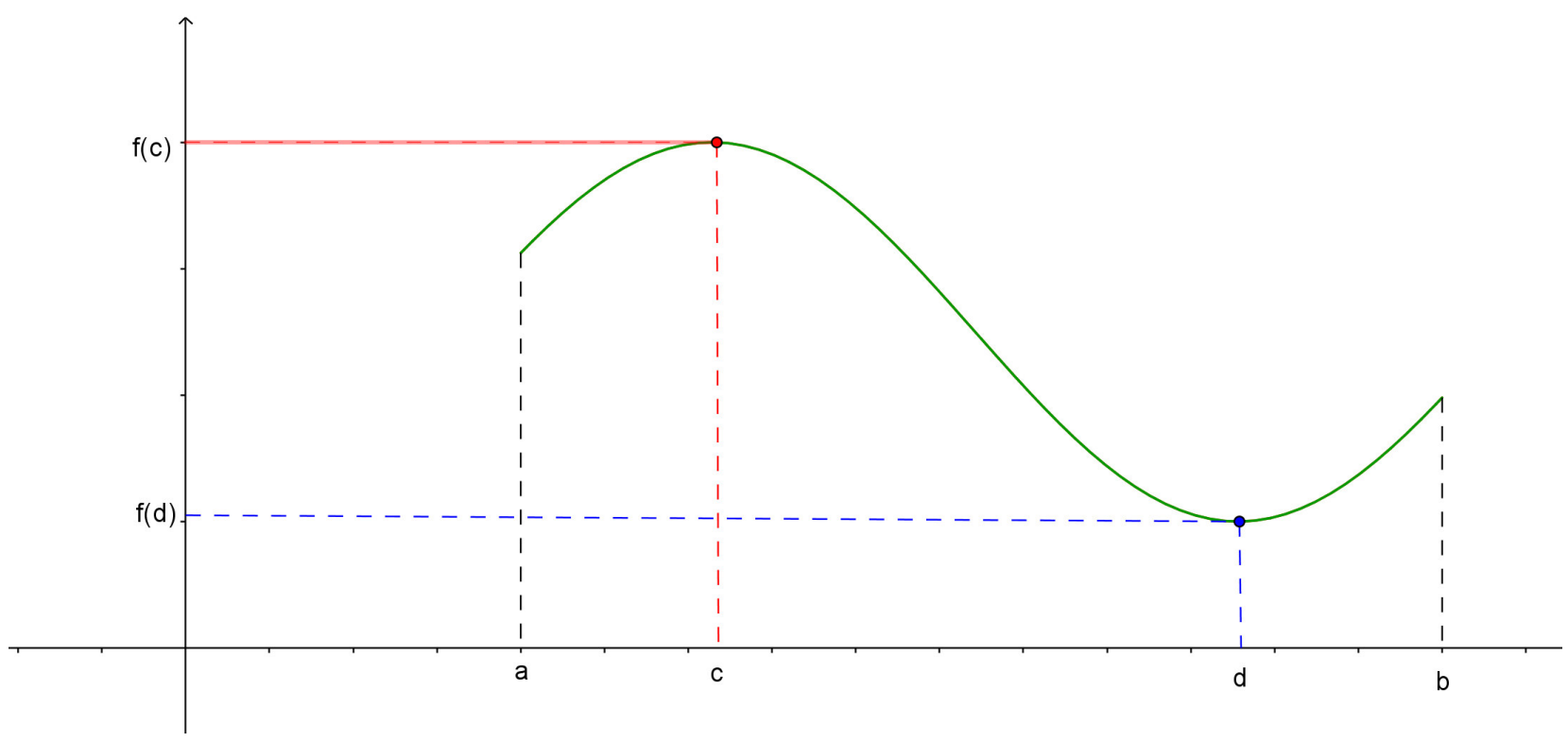

Figura 3.2: Teorema do Valor Extremo

\subsection{O Teorema do Valor Intermediário}

Teorema 3.3.1 (Teorema do Valor Intermediário). Seja uma função $f:[a, b] \rightarrow \mathbb{R}$ contínua em um intervalo fechado $[a, b]$ e d um número qualquer entre $f(a)$ e $f(b)$, onde $f(a) \neq f(b)$. Então existe um número $c \in(a, b)$ tal que $f(c)=d$

Antes de provarmos o Teorema do Valor Intermediário, apresentaremos um resultado que fundamenta a sua demonstração.

Teorema 3.3.2 (Teorema do anulamento). Seja uma função $f:[a, b] \rightarrow \mathbb{R}$ contínua em $[a, b]$ e derivável em $(a, b)$ e se $f(a)$ e $f(b)$ tiverem sinais contrários, então existirá pelo menos um $c \in[a, b]$ tal que $f(c)=0$.

Demonstração. Suponhamos, sem perda de generalidade, que $f(a)<0$ e $f(b)>0$ e considere um conjunto $A=\{x \in[a, b] ; f(x)<0\}$. Como $a \in A, A$ é não vazio e como $A$ está contido em $[a, b], A$ é limitado $\operatorname{logo}, f$ possui um supremo. Seja $c=\sup A$. Então, 
existe $\left(x_{n}\right) \in A$ que converge para $c$. Como $f$ é contínua, então $\lim _{n \rightarrow \infty} f\left(x_{n}\right)=f(c)$. Como $f\left(x_{n}\right)<0$, para todo $n$, então $f(c) \leq 0$.

Por outro lado, se $x>c$, então $f(x) \geq 0$. Tomando-se $\left(y_{n}\right) \subseteq(c, b]$ tal que $\left(y_{n}\right)$ converge para $c$, então $\lim _{n \rightarrow \infty} f\left(y_{n}\right)=f(c) \geq 0$. Logo, $f(c)=0$.

Agora demonstraremos o Teorema do Valor Intermediário.

Demonstração. Suponhamos $f(a)<d<f(b)$. Consideremos a função

$$
g(x)=f(x)-d, x \in[a, b] .
$$

Como $f$ é contínua em $[a, b]$, temos que $g$ também o é. Temos, ainda, que

$$
g(a)=f(a)-d<0 \text { e } g(b)=f(b)-d>0 .
$$

Logo, como g é contínua, existe $c \in[a, b]$ tal que $g(c)=0$, então, $g(c)=f(c)-d$, ou seja, $f(c)=d$.

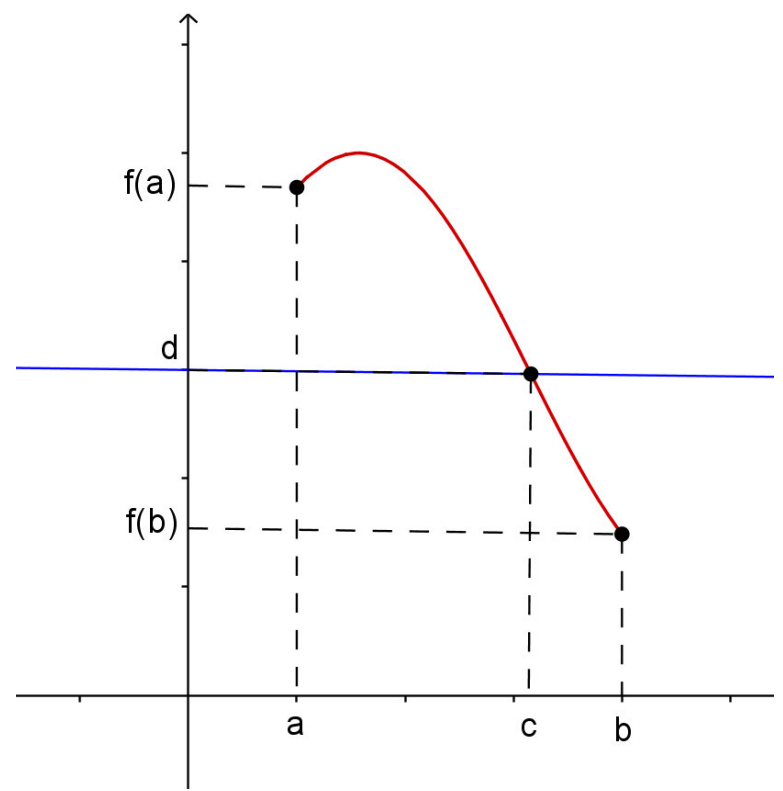

Figura 3.3: Teorema do Valor Intermediário $(f(c)=d)$

O Teorema do Valor Intermediário determina que uma função contínua assume todos os valores intermediários entre os valores assumidos para $f(a)$ e $f(b)$.

Pensando-se em uma função contínua como aquela cujo gráfico não possui falhas nem quebras nem buracos, torna-se fácil admitir a veracidade do Teorema do Valor Intermediário. Em uma visão geométrica, o Teorema estabelece que se for dada uma reta horizontal qualquer $y=d$ entre $f(a)$ e $f(b)$, como na figura 3.3, então o gráfico de $f$ não pode pular sobre a reta, e sim, precisará interceptar $y=d$ em algum ponto. mediário.

Vejamos, a seguir, alguns exemplos da aplicação do Teorema do valor Inter- 
Exemplo: 3.2. Mostre que a equação $x^{3}+x-1=0$ possui uma raiz entre 0 e 1 .

Solução: 3.3.1. Seja $f(x)=x^{3}+x-1$. Queremos encontrar uma solução da equação dada, isto é, um número $c \in(0,1)$ tal que $f(c)=0$. Portanto, tomando $a=0, b=1 \mathrm{e}$ $d=0$ no Teorema do Valor Intermediário, teremos

$$
f(0)=0^{3}+0-1=-1<0 \text { e } f(1)=1^{3}+1-1=1>0 .
$$

Assim, $f(0)<0<f(1)$, ou seja, $d=0$ é um número entre $f(0)$ e $f(1)$. Uma vez que $f$ é contínua por ser polinomial, o Teorema do Valor Intermediário assegura que existe um número $c \in(0,1)$ tal que $f(c)=0$, o que equivale a afirmar que a equação $x^{3}+x-1=0$ tem pelo menos uma raiz $c$ entre 0 e 1 .

Pode-se verificar a existência desta raiz no gráfico da função $f(x)=x^{3}+x-1$, ilustrado na figura 3.4.

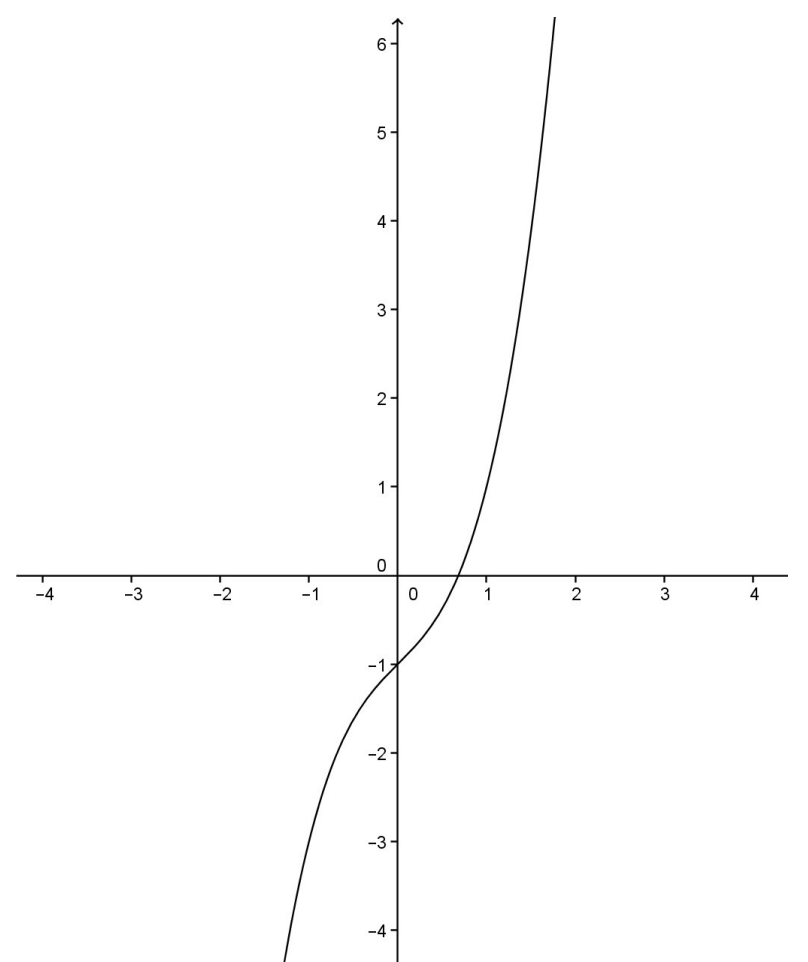

Figura 3.4: Gráfico da função $f(x)=x^{3}+x-1$ 
Exemplo: 3.3. Usando o Teorema do Valor Intermediário, mostre que a equação $x^{2016}+3 x+1=0$ possui alguma raiz negativa.

Solução: 3.3.2. Tomando-se a função $f: \mathbb{R} \rightarrow \mathbb{R}$, definida por $f(x)=x^{2016}+3 x+1$, temos que $f(0)=1$ e $f(-1)=-1$.

Desta forma, como esta função é polinomial, temos que ela é contínua em $[-1,1]$ e, $0 \in[-1,1]$. Então, pelo Teorema do Valor Intermediário, garantimos que existe $c \in(-1,0)$ tal que $f(c)=0$, o que nos leva a concluir que existe um $c$ negativo que é raiz da equação $x^{2016}+3 x+1=0$.

Exemplo: 3.4. Dada a equação $9 x^{4}-10 x^{2}+1=0$.

a) Verifique que esta equação tem pelo menos uma raiz no intervalo $(0,1)$.

Solução: 3.3.3. Seja $f(x)=9 x^{4}-10 x^{2}+1$, a função $f$ é uma função polinomial e, portanto, $f$ é uma função contínua em $\mathbb{R}$.

Como $[0,1] \in \operatorname{Dom}(f)=\mathbb{R}$, temos que $f$ é contínua em $[0,1]$. Sabemos que $(0 ; 0,9) \subset(0,1)$ e, ainda por ser polinomial, $f$ é contínua em $[0 ; 0,9]$. Sendo

$$
f(0)=9 \cdot 0^{4}-10 \cdot 0^{2}+1=1>0 \text { e } f(0,9)=9 \cdot(0,9)^{4}-10 \cdot(0,9)^{2}+1=-1,1951<0,
$$

então, pelo Teorema do Valor Intermediário, existe $c \in(0 ; 0,9)$ tal que $f(c)=0$, o que nos permite afirmar que existe $c \in(0,1)$ tal que $f(c)=0$, pois $(0 ; 0,9) \subset(0,1)$. Logo, existe pelo menos uma solução da equação $9 x^{4}-10 x^{2}+1=0$ no intervalo $(0,1)$.

b) Observe, abaixo, no gráfico da função $f(x)=9 x^{4}-10 x^{2}+1$, que este polinômio possui 4 raízes reais.

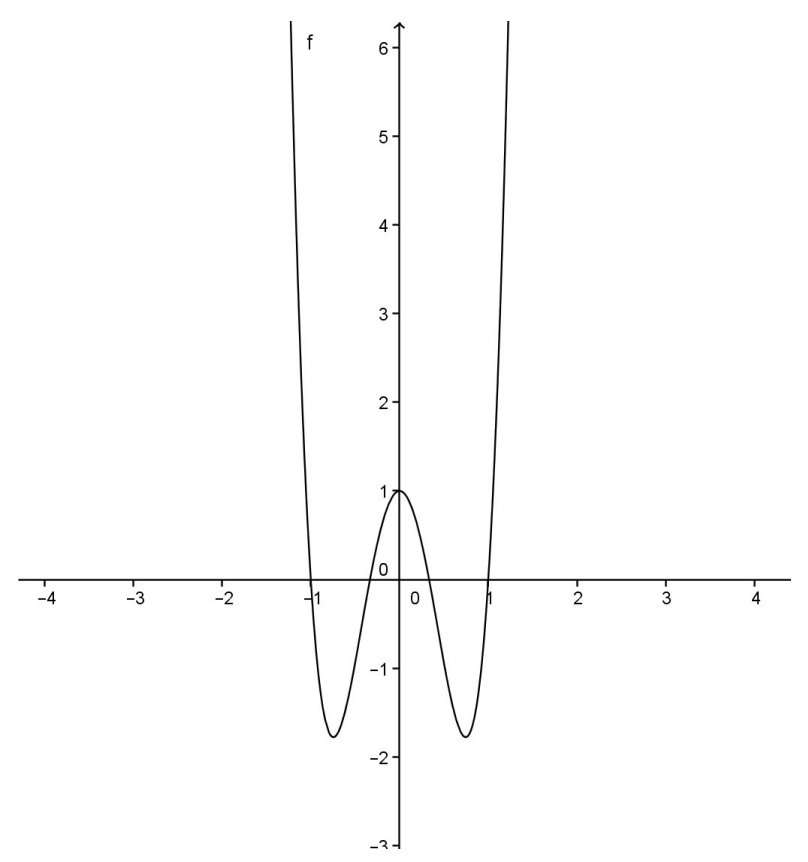

Figura 3.5: Gráfico da função $f(x)=9 x^{4}-10 x^{2}+1$ 
Verifique que esta função é par e que -1 e 1 são raízes de $f$. Além disso, veja neste gráfico que as outras raízes estão no intervalo $(0,1)$ (conforme pedido para verificar no item $a)$ ) e no intervalo $(-1,0)$.

\section{Solução: 3.3.4.}

(i) A função $f(x)=9 x^{4}-10 x^{2}+1$ é uma função polinomial de grau 4 e vemos em seu gráfico que ela possui 4 intersecções com o eixo $x$, logo possui 4 raízes.

(ii) Observe que, pelo menos no intervalo representado neste gráfico, temos uma simetria em relação ao eixo $y$. Temos que:

(1) $\operatorname{Dom}(f)=\mathbb{R}, \log 0-x \in \operatorname{Dom}(f)$ sempre que $x \in \operatorname{Dom}(f)$;

(2) $f(-x)=9(-x)^{4}-10(-x)^{2}+1=9 x^{4}-10 x^{2}+1=f(x), \forall x \in \operatorname{Dom}(f)=\mathbb{R}$.

Então, por (1) e (2) concluímos que $f$ é uma função par.

(iii) $f(-1)=9(-1)^{4}-10(-1)^{2}+1=0$ e $f(1)=9 \cdot 1^{4}-10 \cdot 1^{2}+1=0$.

Logo, -1 e 1 são raízes de $f$.

(iv) Já sabemos que -1 e 1 são raízes de $f$ e que $f$ possui 4 raízes reais. Vemos no gráfico de $f$ que nos intervalos $(-1,0)$ e $(0,1)$ existem intersecções com o eixo $x$, logo as outras duas raízes estão nestes intervalos.

(c) Calculando um valor aproximado de f para alguns valores de x encontramos:

\begin{tabular}{|c|c|c|c|c|c|c|c|c|c|}
\hline$x$ & $-0,9$ & $-0,8$ & $-0,7$ & $-0,6$ & $-0,5$ & $-0,4$ & $-0,3$ & $-0,2$ & $-0,1$ \\
\hline$f(x)$ & $-1,1951$ & $-1,7136$ & $-1,7391$ & $-1,4336$ & $-0,9375$ & $-0,3696$ & 0,1729 & 0,6144 & 0,9009 \\
\hline
\end{tabular}

Com estas informações, lembrando que -1 e 1 são raízes de $f$, quais são os menores intervalos abertos onde podemos encontrar as outras duas raízes? Por que?

Solução: 3.3.5. Com as informações dadas, vemos que os menores intervalos onde podemos encontrar as outras duas raízes, são $(-0,4 ;-0,3)$ e $(0,3 ; 0,4)$. O porquê de tal afirmação se deve ao fato de, sendo $f$ uma função polinomial e, portanto, contínua em $\mathbb{R}$, então suas raízes podem ser identificadas ao observar-se a mudança de sinais dos valores que a função admite, de positivo para negativo ou de negativo para positivo. Como $f$ é uma função par, então

$$
f(0,3)=f(-0,3)=0,1729>0 \text { e } f(0,4)=f(-0,4)=-0,3696<0 .
$$

d) Demonstre formalmente que essas outras duas raízes realmente se encontram nos intervalos encontrados no item c.

Demonstração. Temos que:

(i) $f$ é contínua em $[-0,4 ;-0,3]$;

(ii) $f(-0,4) \cong-0,3696<0$ e $f(-0,3) \cong 0,1729>0$.

Então, pelo Teorema do valor Intermediário, existe $c \in(-0,4 ;-0,3)$ tal que $f(c)=0$.

Portanto, $f$ possui pelo menos uma raiz no intervalo $(-0,4 ;-0,3)$ e, como $f$ é uma função par, temos que $f$ também possui pelo menos uma raiz no intervalo $(0,3 ; 0,4)$. 
Exemplo: 3.5. Um alpinista inicia a escalada de uma montanha às 6 h e chega ao cume às $18 h$, passa a noite descansando, e inicia a decida às $6 h$ do dia seguinte, chegando de volta à base da montanha às $18 h$. Prove que existe um ponto do trajeto em que o alpinista estará, na mesma hora do dia, tanto na subida quanto na descida.

Demonstração. Consideremos as seguintes funções contínuas,

$$
s:[6,18] \rightarrow \mathbb{R} \text { e } d:[6,18] \rightarrow \mathbb{R},
$$

as quais nomearemos, respectivamente, por função subida e função descida. Assim, $s(t)$ representa a posição do alpinista no instante $t$ durante a subida, enquanto que $d(t)$ representa a posição do alpinista no instante $t$ durante a descida. Tomemos uma função contínua $f:[6,18] \rightarrow \mathbb{R}$ definida por

$$
f(t)=s(t)-d(t)
$$

Podemos considerar, sem perda de generalidade, 0 (zero) como a posição na base da montanha e $C$ a posição no cume da montanha. Assim,

$$
s(6)=0, s(18)=C, d(6)=C \text { e } d(18)=0 .
$$

Desta forma,

$$
f(6)=s(6)-d(6)=0-C=-C<0 \text { e } f(18)=s(18)-d(18)=C-0=C>0 .
$$

Portanto, pelo Teorema do Valor Intermediário, existe $t_{0} \in(6,18)$ tal que $f\left(t_{0}\right)=0$, então $s\left(t_{0}\right)-d\left(t_{0}\right)=0$, ou seja, $s\left(t_{0}\right)=d\left(t_{0}\right)$. 


\section{O TEOREMA DE ROLLE}

\subsection{O Teorema de Rolle}

Importante resultado publicado pelo matemático francês Michel Rolle (16521719) no livro Méthode pour résoudre les égalitéz, em 1691. Consiste em determinar os pontos do gráfico de uma função contínua e derivável em um dado intervalo em que a reta tangente possui inclinação igual a 0 (zero). Vejamos, a seguir, o que enuncia o teorema.

Teorema 4.1.1 (Teorema de Rolle). Seja uma função $f:[a, b] \rightarrow \mathbb{R}$ contínua em $[a, b]$ e derivável em $(a, b)$ com $f(a)=f(b)$. Então, existe um ponto $c \in(a, b)$ tal que $f^{\prime}(c)=0$.

Demonstração. Como $f$ é contínua, pelo Teorema do Valor Extremo, $f$ assume um valor máximo absoluto e um valor mínimo absoluto em $[a, b]$.

Sejam $M$ e $m$, respectivamente o máximo absoluto e o mínimo absoluto de $f$. Então, se $f$ for constante, teremos $M=n=f(a)=f(b)$, e consequentemente $f^{\prime}(x)=0$, para todo $x \in[a, b]$. Logo, qualquer número entre $a$ e $b$ pode ser tomado para $c$.

Suponhamos agora que $M \neq m$. Então, como $f(a)=f(b)$ devemos ter $M=f(c)$, para algum $c$ tal que $a<c<b$. Então para $x<c$ teremos $x-c<0$ e também $f(x)-f(c) \leq 0$ e, portanto,

$$
\frac{f(x)-f(c)}{x-c} \geq 0
$$

Como $f$ é derivável em $(a, b)$, temos que

$$
\lim _{x \rightarrow c^{-}} \frac{f(x)-f(c)}{x-c}=f^{\prime}(c) \geq 0 .
$$

Como $f$ é derivável em $(a, b)$, segue que

$$
\lim _{x \rightarrow c^{-}} \frac{f(x)-f(c)}{x-c}=f^{\prime}(c) \geq 0 .
$$

Do mesmo modo, se $x>c$, então $x-c>0$, e também $f(x)-f(c) \leq 0$, e assim,

$$
\frac{f(x)-f(c)}{x-c} \leq 0
$$

. Daí, temos

$$
\lim _{x \rightarrow c^{+}} \frac{f(x)-f(c)}{x-c}=f^{\prime}(c) \leq 0 .
$$

Portanto, como $f^{\prime}(c) \geq 0$ e $f^{\prime}(c) \leq 0$, a única possibilidade é $f^{\prime}(c)=0$.

Os gráficos a seguir exemplificam funções que cumprem os requisitos que possibilitam a aplicação do Teorema de Rolle, ou seja, a determinação do(s) ponto(s) do gráfico de uma função em que a tangente ao gráfico é uma reta horizontal e, portanto, $f^{\prime}(c)=0$. 


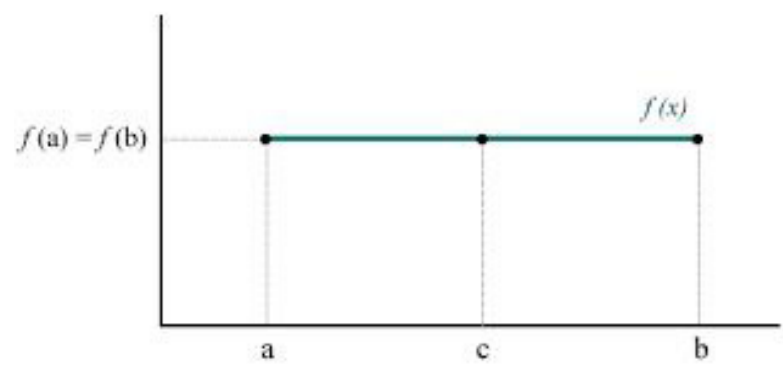

Figura 4.1: Teorema de Rolle (função constante)

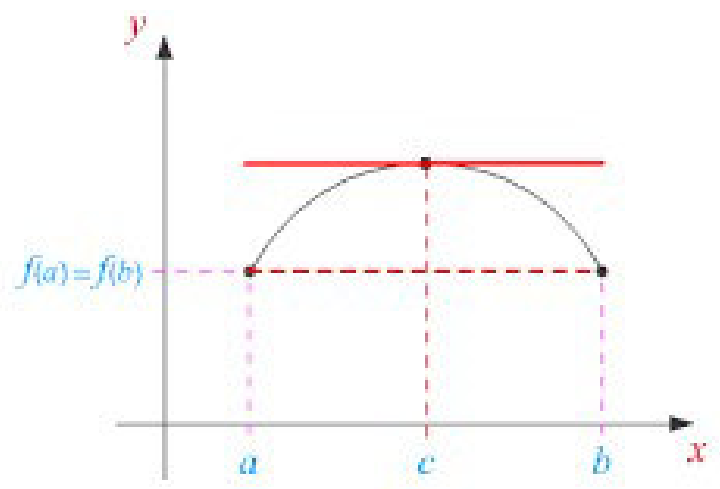

Figura 4.2: Teorema de Rolle (função polinomial do $2^{\circ}$ grau, $a<0$

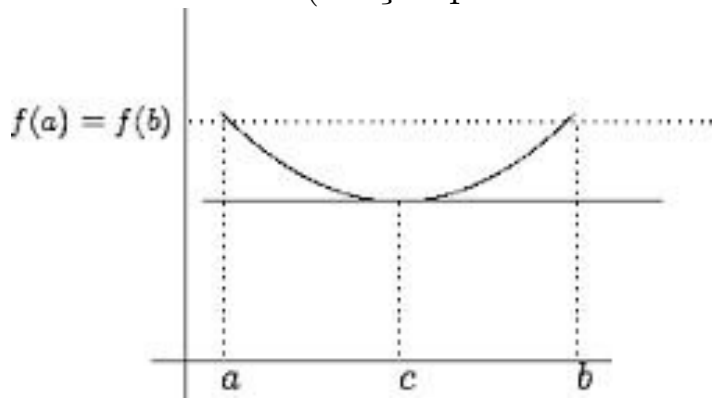

Figura 4.3: Teorema de Rolle (função polinomial do $2^{\circ}$ grau, $a>0$

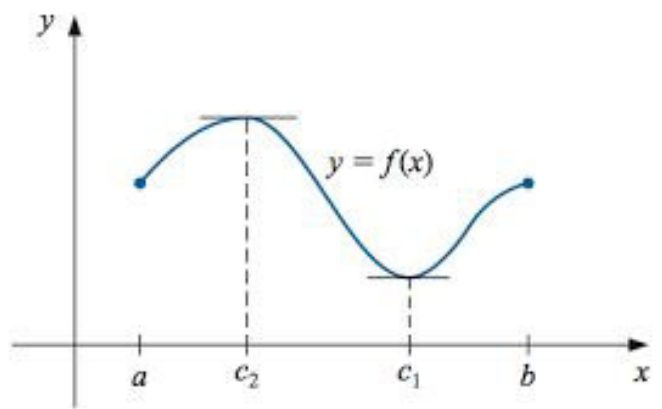

Figura 4.4: Teorema de Rolle (função polinomial em $[a, b]$ )

Exemplo: 4.1. Considere a função $s=f(t)$, onde $s$ representa a posição de um objeto no instante $t$. Tendo estado o objeto na mesma posição em dois instantes diferentes $t=a$ e $t=b$, ou seja, $s(a)=s(b)$, pelo Teorema de Rolle podemos afirmar que existe algum instante $t=c, c \in(a, b)$, onde $s^{\prime}(c)=0$, o que equivale a dizer que no instante $t=c$ a velocidade é 0. (Em particular, essa afirmação é facilmente verificada quando algum corpo é atirado diretamente para cima.) 
Exemplo: 4.2. Prove que a equação $x^{3}+2 x-1=0$ tem exatamente uma raiz real.

Demonstração. Aplicamos, inicialmente, o Teorema do Valor Intermediário para verificar a existência de uma raiz. Seja $f(x)=x^{3}+2 x-1$. Então, $f(0)=-1<0$ e $f(1)=2>0$. Como $f$ é uma função polinomial, ela é contínua; logo, o Teorema do Valor Intermediário garante a existência de um número $c \in(0,1)$ tal que $f(c)=0$. Portanto, a equação dada tem uma raiz.

Para mostrar que esta raiz é única, aplicamos o Teorema de Rolle e demonstramos por contradição. Suponhamos que existam duas raízes $a$ e $b$, da a equação dada. Então, $f(a)=f(b)=0$ e, como $f$ é uma função polinomial, é contínua em $[a, b]$ e derivável em $(a, b)$. Logo, pelo Teorema de Rolle, existe um número $c \in(a, b)$ tal que $f^{\prime}(c)=0$. Mas $f^{\prime}(x)=3 x^{2}+2 \geq 2$ para todo $x$, pois $x^{2} \geq 0$, portanto, $f^{\prime}(x)$ nunca pode ser zero. Isso nos traz a uma contradição. Por conseguinte, a equação não pode ter duas raízes.

Exemplo: 4.3. Considere a função $f$ definida por $f(x)=x(x-1)^{2016}$. Usando o Teorema de Rolle verifique que existe $c \in(0,1)$ tal que a reta tangente ao gráfico de $f$ no ponto $(c, f(c))$ é paralela ao eixo $x$. Determine o valor de $c$.

Solução: 4.1.1. Temos que:

1. $f(x)=x(x-1)^{2016}$ é uma função polinomial e, portanto, $f$ é contínua em $\operatorname{Dom}(f)=\mathbb{R}$. Em particular, $f$ é contínua em $[0,1]$;

2.

$$
\begin{aligned}
f^{\prime}(x)=(x-1)^{2016}+x \cdot 2016(x-1)^{2015} \cdot 1 & =(x-1)^{2016}+2016 x(x-1)^{2015} \\
& =(x-1)^{2015}((x-1)+2016 x) \\
& =(x-1)^{2015}(2017 x-1)
\end{aligned}
$$

para todo $x \in \mathbb{R}$. Em particular, $f$ é derivável em $(0,1)$;

3. $f(0)=0 \cdot(0-1)^{2016}=0$ e $f(1)=1 \cdot(1-1)^{2016}=0$.

Portanto, pelo Teorema de Rolle, existe $c \in(0,1)$ tal que $f^{\prime}(c)=0$.

Se $f^{\prime}(c)=0$, então $(c-1)^{2015}(2017 c-1)=0$, ou ainda,

$$
\left\{\begin{array}{c}
(c-1)^{2015}=0, \text { o que resulta em } c=1 \notin(0,1) \\
2017 c-1=0, \text { o que resulta em } c=\frac{1}{2017} \in(0,1)
\end{array} .\right.
$$

Exemplo: 4.4. Mostre que a equação $x^{3}+x+c=0, c \in \mathbb{R}$, não pode ter mais de uma raiz real.

Demonstração. Seja $f: \mathbb{R} \rightarrow \mathbb{R}$ uma função definida por $f(x)=x^{3}+x+c$. Suponhamos que $f$ possua duas raizes reais $r_{1}$ e $r_{2}$. $f$ é polinomial e, portanto, $f$ é contínua e derivável em $\mathbb{R}$, em particular, $f$ é contínua em $\left[r_{1}, r_{2}\right]$ e derivável em $\left(r_{1}, r_{2}\right)$. Suponhamos que $f$ tenha duas raízes reais, $r_{1}$ e $r_{2}$, ou seja, $f\left(r_{1}\right)=f\left(r_{2}\right)=0$, com $r_{1}<r_{2}$. Então, pelo Teorema de Rolle, existe $c \in\left(r_{1}, r_{2}\right)$ tal que $f^{\prime}(c)=0$. Logo, como $f^{\prime}(x)=3 x^{2}+1 \neq 0$, para todo $x \in \mathbb{R}$, então concluímos que $f$ não pode ter mais de uma raiz real. 


\section{UMA APLICAÇÃO PARTICULAR DO TEOREMA DE ROLLE}

Neste capítulo apresentaremos uma aplicação particular do Teorema de Rolle que se consiste em sua restrição à um caso particular.

\subsection{Uma aplicação Particular do Teorema de Rolle}

Da aplicação do Teorema de Rolle que acabamos de ver, no capítulo anterior, vejamos o seguinte exemplo.

Exemplo: 5.1. Dada a função $f(x)=2 x^{2}-8$ contínua em $[-2,2]$ e drivável em $(-2,2)$, determinar $c \in(-2,2)$ tal que $f^{\prime}(c)=0$.

Solução: 5.1.1. Temos que $f$ é contínua em $[-2,2]$ e derivável em $(-2,2)$ e, ainda, $f(-2)=f(2)=0$. Logo, pelo Teorema de Rolle, deve existir $c \in(-2,2)$ tal que $f^{\prime}(c)=0$. Visto que $f^{\prime}(x)=4 x$, temos $f^{\prime}(x)=0, \operatorname{logo}, x=0$, e portanto, vemos que $c=0$.

Vejamos, agora, o seguinte caso particular.

Seja $g(x)=2 x^{2}-8$ e $f(x)=g(x) e^{-2 x}$. Ainda temos $f$ contínua em $[-2,2]$, derivável em $(-2,2)$ e $f(-2)=f(2)=0$. Mas,

$$
f^{\prime}(x)=g^{\prime}(x) e^{-2 x}-2 g(x) e^{-2 x}=\left[g^{\prime}(x)-2 g(x)\right] e^{-2 x} .
$$

Pelo Teorema de Rolle, existe $c \in(-2,2)$ tal que $f^{\prime}(c)=0$. Como $e^{-2 x} \neq 0$, para todo $x \in \mathbb{R}$, temos que $c$ deve ser a raiz de

$$
g^{\prime}(c)-2 g(c)=0 \text {, ou seja, } g^{\prime}(c)=2 g(c) .
$$

A interpretação gráfica deste resultado é que, entre os pontos $(-2, g(-2))$ e $(2, g(2))$ do gráfico de $g$, há pelo menos um ponto cuja tangente tem declividade igual ao dobro do valor de $g$ nesse ponto. Para determinação desse ponto, teremos

$g^{\prime}(c)-2 g(c)=0$, o que resulta em $4 c-2\left(2 c^{2}-8\right)=0$, ou melhor, $-c^{2}+c+4=0$, e assim, teremos $c=\frac{1 \pm \sqrt{17}}{2}$.

Porém, c deve estar em $(-2,2)$, então $c=\frac{1-\sqrt{17}}{2}$. 


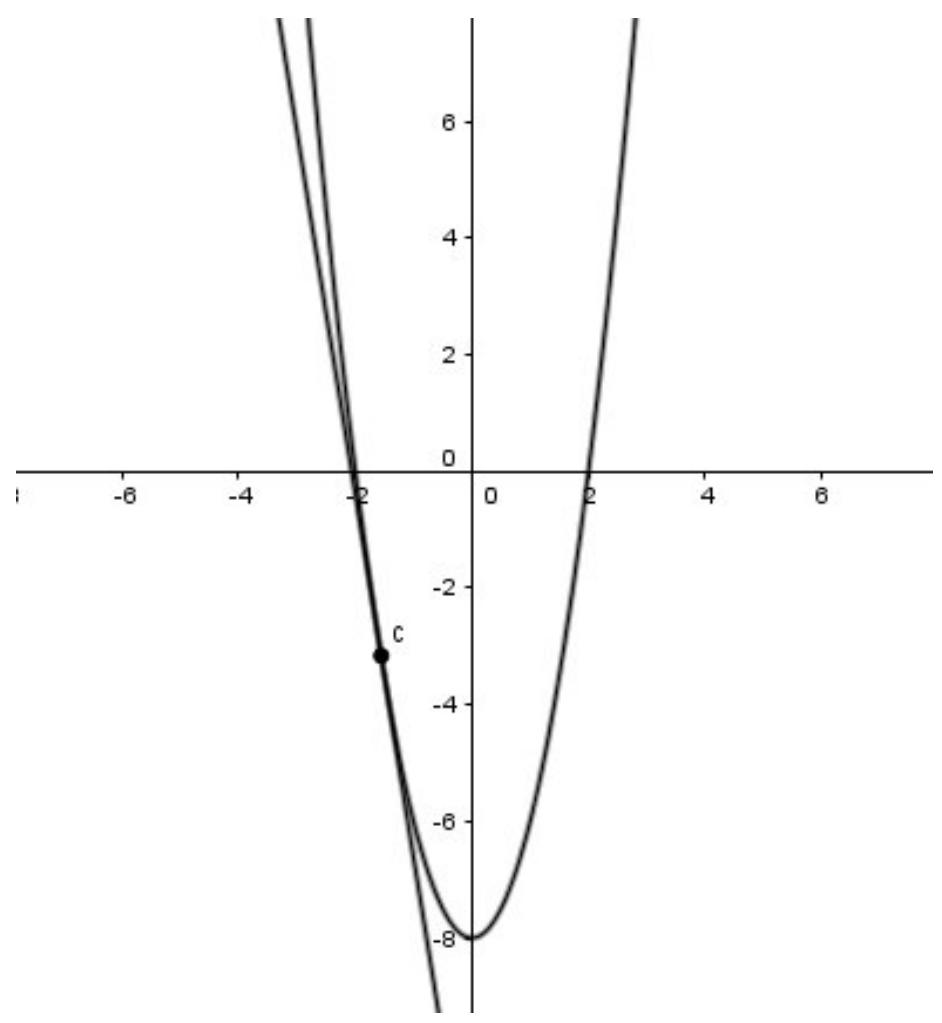

Figura 5.1: Teorema de Rolle (Reta tangente à curva $y=2 x^{2}-8$ em $c=\frac{1-\sqrt{17}}{2}$

Por generalização, se $g(x)=2 x^{2}-8$ e tomarmos $f(x)=g(x) e^{-k x}, k \in \mathbb{R}$, obtemos $c \in(-2,2)$ tal que

$$
g^{\prime}(c)-k g(c)=0
$$

Com base neste resultado, enunciamos o seguinte teorema formalizando esta aplicação particular do Teorema de Rolle.

Teorema 5.1.1. Seja uma função $g:[a, b] \rightarrow \mathbb{R}$ contínua em $[a, b]$ e derivável em $(a, b)$. Se $g(a)=g(b)=0$, então para todo $k \in \mathbb{R}$, existe $c \in(a, b)$ tal que

$$
g^{\prime}(c)=k g(c)
$$

Observação: 5.1.1. Como uma aplicação particular do teorema de Rolle, este teorema está relacionado à inclinação da reta tangente à uma curva qualquer, observando-se assim que uma das condições necessárias é que a função que define a curva seja diferenciável apenas no intervalo aberto $(a, b)$, o que equivale a afirmar que a função tem que ser diferenciável apenas nos pontos interiores do intervalo $[a, b]$, ou seja, diferenciável em int $[a, b]$.

Demonstração. Seja $g$ uma função contínua em $[a, b]$ e derivável em $(a, b)$. Tomemos, arbitrariamente, um número real $k$ e uma função $f$, definida por $f(x)=g(x) e^{-k x}$. Temos que $f$ é contínua em $[a, b]$, pois é o produto de duas funções contínuas em $[a, b]$, conforme o item (iii) da proposição 2.5, e $f$ também é derivável em $(a, b)$, pois é um produto de funções deriváveis em $(a, b)$, conforme o item $(v)$ da subseção 2.4.6. Como $e^{-k x} \neq 0$, para todo $x \in \mathbb{R}$, as raízes de $f(x)$ são as mesmas de $g(x)$, o que nos permite afirmar que 
$f(a)=f(b)=0$. Vemos, assim, que a função $f$ satisfaz as condições de aplicação do Teorema de Rolle. Então, existe um número $c \in(a, b)$, tal que $f^{\prime}(c)=0$. Mas,

$$
f^{\prime}(x)=g^{\prime}(x) e^{-k x}-k g(x) e^{-k x}
$$

daí, temos

$$
f^{\prime}(c)=g^{\prime}(c) e^{-k c}-k g(c) e^{-k c}=\left(g^{\prime}(c)-k g(c)\right) e^{-k c}
$$

de onde se conclui que, analogamente ao que decorre das raízes de $g(x)$ e $f(x)$, existe pelo menos uma raiz de $f^{\prime}(c)$, o que verifica-se quando

$g^{\prime}(c)-k g(c)=0$, ou seja, $g^{\prime}(c)=k g(c)$, o que comprova a afirmação previamente enunciada.

O fato de termos tomado $k$ arbitrariamente nos possibilita afirmar que, para todo $k \in \mathbb{R}$ existe um número $c \in(a, b)$ tal que $f^{\prime}(c)=k f(c)$, sem explicitarmos a generalidade da relação entre $c$ e $k$. Geometricamente, podemos visualizar este resultado como a garantia de que no gráfico de uma função $g$, que satisfaça as condições de aplicação do Teorema de Rolle, existe pelo menos um ponto entre $(a, g(a))$ e $(b, g(b))$ cuja tangente possui declividade igual a um múltiplo arbitrário do valor admitido por $g$ nesse ponto.

Exemplo: 5.2. Verifique se existe algum ponto do gráfico da função $g: \mathbb{R} \rightarrow \mathbb{R}$ definida por $g(x)=x^{2}-3 x+2$ onde a sua derivada é igual ao quíntuplo do valor da função, neste mesmo ponto. Caso exista, determine este ponto.

Solução: 5.1.2. Os zeros desta função são $x=1$ e $x=2$, ou seja $g(1)=g(2)=0$. Por ser polinomial, temos que a função $g$ é contínua e derivável em $\mathbb{R}$; em particular, temos que $g$ é contínua em $[1,2]$ e derivável em $(1,2)$. Logo, pelo Teorema de Rolle, deve existir $c \in(1,2)$ tal que $g^{\prime}(c)=0$. Visto que $g^{\prime}(x)=2 x-3$, temos $g^{\prime}(x)=0$, de onde se tem $x=\frac{3}{2}$ e, portanto, vemos que $c=\frac{3}{2}$.

Tomemos, agora, uma função $f: \mathbb{R} \rightarrow \mathbb{R}$ definida por $f(x)=g(x) e^{-5 x}$. Temos que $f$ é contínua em $[1,2]$, derivável em $(1,2)$ e $f(1)=f(2)=0$. Mas,

$$
f^{\prime}(x)=g^{\prime}(x) e^{-5 x}-5 g(x) e^{-5 x}=\left[g^{\prime}(x)-5 g(x)\right] e^{-5 x} .
$$

Pelo Teorema de Rolle, existe $c \in(1,2)$ tal que $f^{\prime}(c)=0$. Como $e^{-5 x} \neq 0$, para todo $x \in \mathbb{R}$, temos que $c$ deve ser a raiz de

$$
g^{\prime}(c)-5 g(c)=0 \text {, ou seja, } g^{\prime}(c)=5 g(c)
$$

Interpretando graficamente este resultado, temos que, entre os pontos $(1, g(1))$ e $(2, g(2))$ do gráfico de $g$, há pelo menos um ponto cuja tangente tem declividade igual ao quíntuplo do valor de $g$ nesse ponto. Para determinação desse ponto, teremos $g^{\prime}(c)-5 g(c)=0$, isto é, $(2 c-3)-5\left(c^{2}-3 c+2\right)=0$ o que resulta em $c=\frac{17 \pm \sqrt{29}}{10}$. Mas, devemos ter $c \in(1,2)$, então, $c=\frac{17-\sqrt{29}}{10}$. 


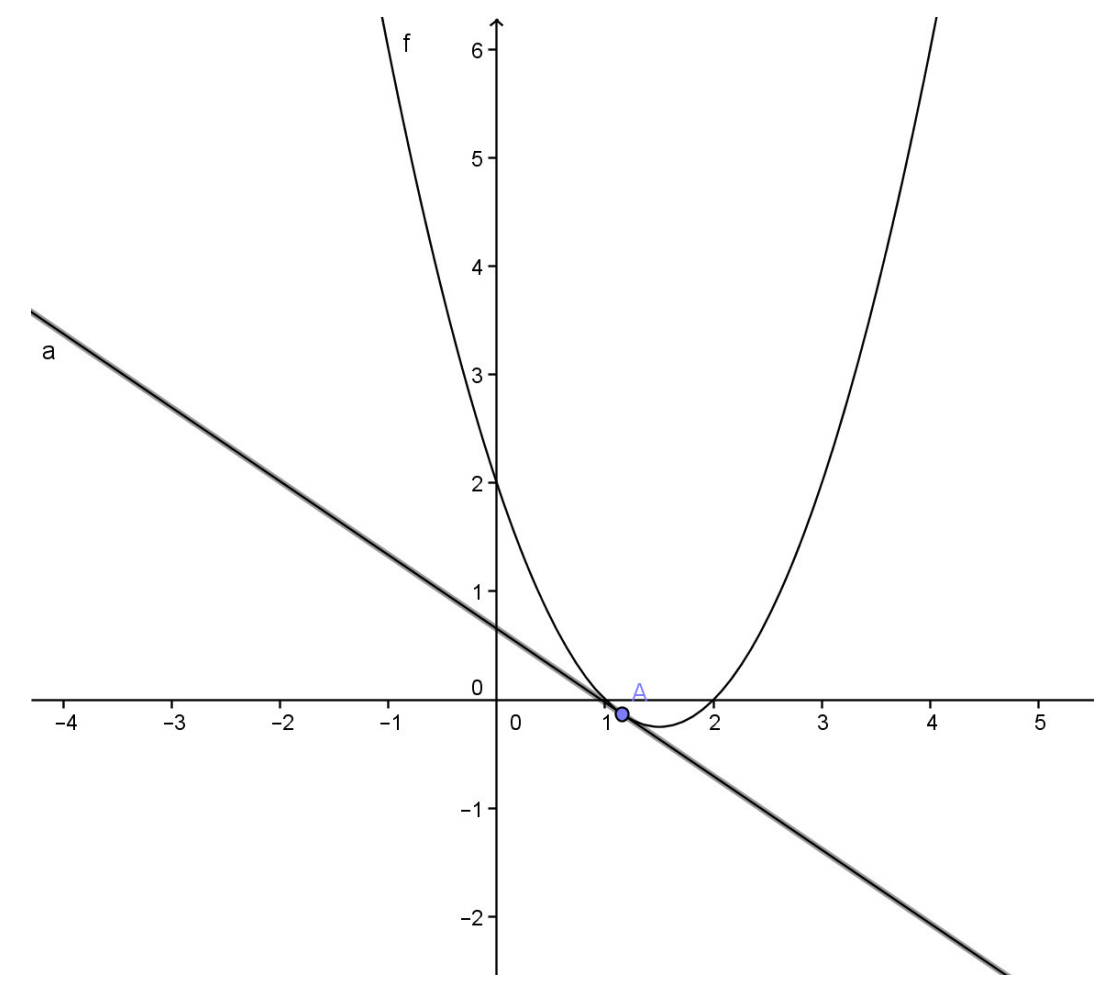

Figura 5.2: Teorema de Rolle tangente à curva $y=x^{2}-3 x+2$ em $c=\frac{17-\sqrt{29}}{10}$

Exemplo: 5.3. Determine, caso exista, o ponto do gráfico da função $g: \mathbb{R} \rightarrow \mathbb{R}$ definida por $g(x)=x^{2}-1$ onde a sua derivada é igual à metade do valor da função, neste mesmo ponto.

Solução: 5.1.3. Temos que $g(-1)=g(1)=0$ e, por ser polinomial, temos que a função $g$ é contínua e derivável em $\mathbb{R}$; em particular, temos que $g$ é contínua em $[-1,1]$ e derivável em $(-1,1)$. Logo, pelo Teorema de Rolle, deve existir $c \in(-1,1)$ tal que $g^{\prime}(c)=0$. Visto que $g^{\prime}(x)=2 x$, temos $g^{\prime}(x)=0, \operatorname{logo} x=0$, e portanto, vemos que $c=0$.

Tomemos, agora, uma função $f: \mathbb{R} \rightarrow \mathbb{R}$ definida por $f(x)=g(x) e^{-\frac{1}{2} x}$. Temos que $f$ é contínua em $[-1,1]$, derivável em $(-1,1)$ e $f(-1)=f(1)=0$. Mas,

$$
f^{\prime}(x)=g^{\prime}(x) e^{-\frac{1}{2} x}-\frac{1}{2} g(x) e^{-\frac{1}{2} x}=\left[g^{\prime}(x)-\frac{1}{2} g(x)\right] e^{-\frac{1}{2} x} .
$$

Pelo Teorema de Rolle, existe $c \in(-1,1)$ tal que $f^{\prime}(c)=0$. Como $e^{-\frac{1}{2} x} \neq 0$, para todo $x \in \mathbb{R}$, temos que $c$ deve ser a raiz de

$$
g^{\prime}(c)-\frac{1}{2} g(c)=0, \text { isto é, } g^{\prime}(c)=\frac{1}{2} g(c)
$$

Interpretando graficamente este resultado, temos que, entre os pontos $(-1, g(-1))$ e $(1, g(1))$ do gráfico de $g$, há pelo menos um ponto cuja tangente tem declividade igual à metade do valor de $g$ nesse ponto. Para determinação desse ponto, teremos $g^{\prime}(c)-\frac{1}{2} g(c)=0$, ou seja, $2 c-\frac{1}{2}\left(c^{2}-1\right)=0$, o que resulta em $c=2 \pm \sqrt{5}$.

Contudo, devemos ter $c \in(-1,1)$, então, $c=2-\sqrt{5}$. 


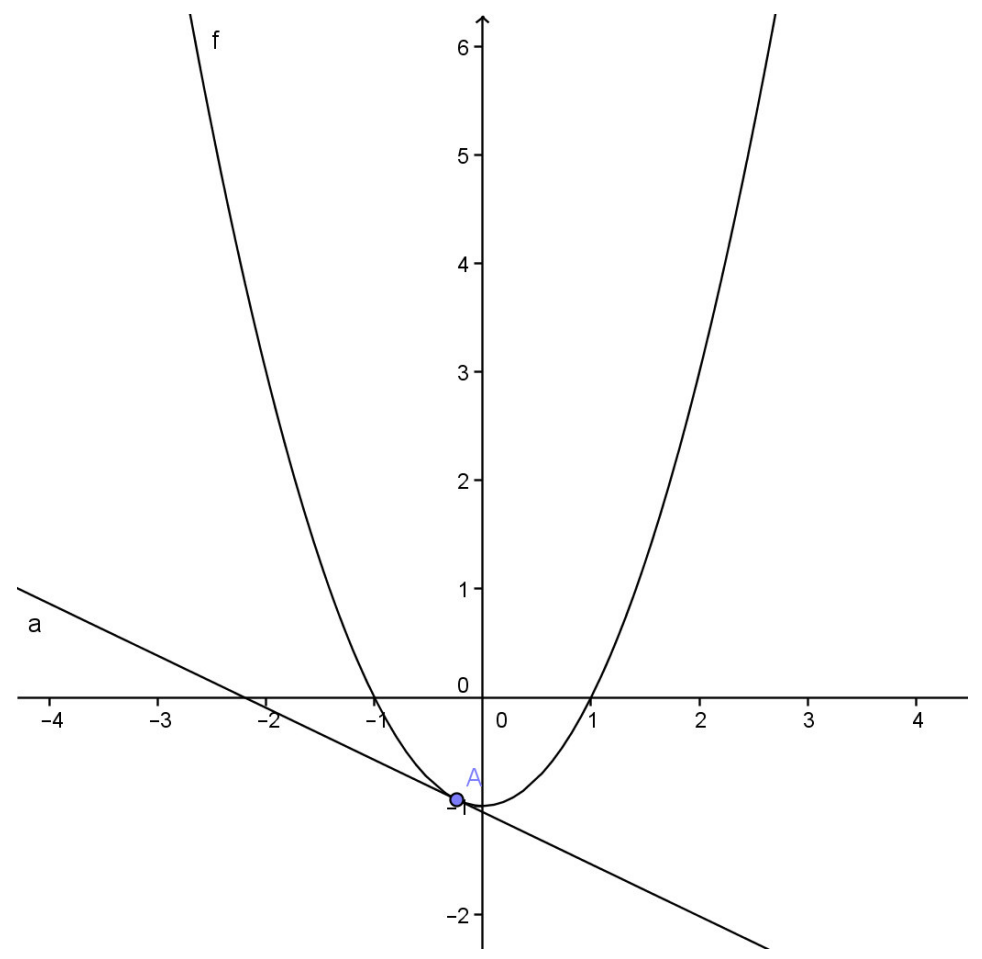

Figura 5.3: Teorema de Rolle (Reta tangente à curva $y=x^{2}-1$ em $c=2-\sqrt{5}$ ) 


\section{O TEOREMA DO VALOR MÉDIO}

\subsection{O Teorema do Valor Médio}

Teorema 6.1.1 (Teorema do Valor Médio). Seja uma função $f:[a, b] \rightarrow \mathbb{R}$ contínua em $[a, b]$ e derivável em $(a, b)$. Então existe um número $c \in(a, b)$ tal que:

$$
f^{\prime}(c)=\frac{f(b)-f(a)}{b-a}
$$

A interpretação geométrica deste teorema estabelece que, se uma função $y=f(x)$ é contínua em $[a, b]$ e derivável em $(a, b)$, então existe pelo menos um ponto $c$ entre os pontos $a$ e $b$ onde a reta tangente à curva é paralela à reta suporte da corda que une os pontos $P(a, f(a))$ e $Q(b, f(b))$, conforme a figura abaixo.

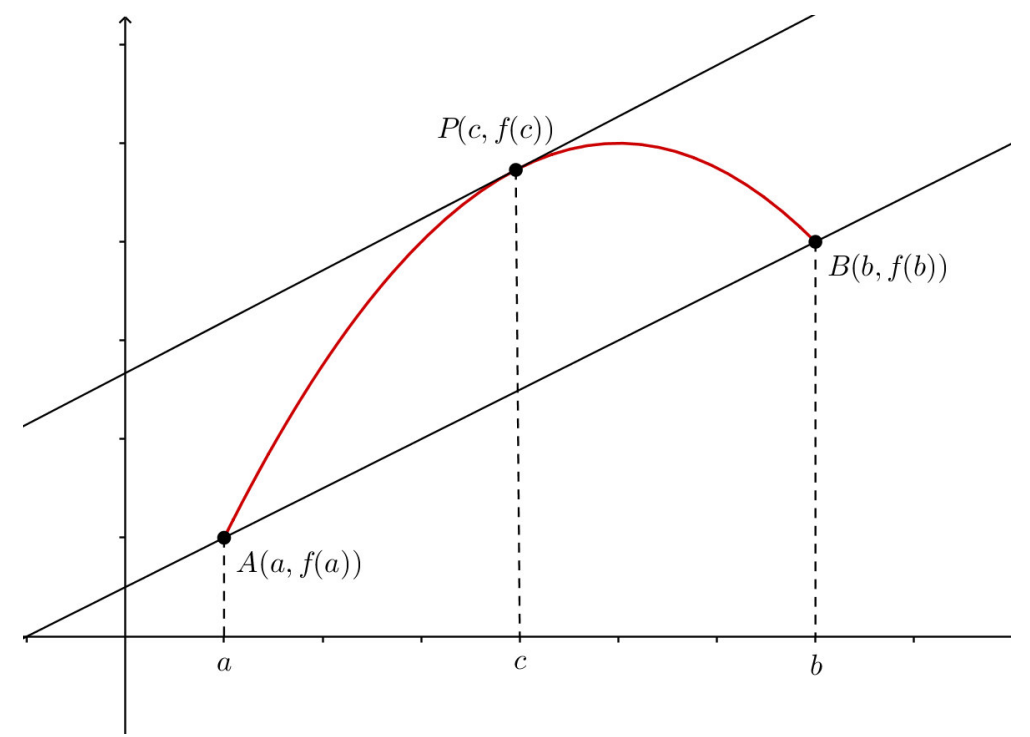

Figura 6.1: Tangente à curva $y=f(x)$ paralela à reta secante $P Q$

Observação: 6.1.1. Como o teorema do Valor Médio está relacionado à inclinação da reta tangente à uma curva qualquer, vê-se que uma das condições necessárias é que a função que define a curva seja diferenciável apenas no intervalo aberto $(a, b)$, o que equivale a afirmar que a função tem que ser diferenciável apenas nos pontos interiores do intervalo $[a, b]$, ou seja, diferenciável em int $[a, b]$.

Demonstração. Dados $P(a, f(a))$ e $Q(b, f(b))$. A equação da reta $\stackrel{\leftrightarrow}{P Q}$ é

$$
y-f(a)=\frac{f(b)-f(a)}{b-a}(x-a) .
$$

Fazendo $y=h(x)$, teremos $h(x)=\frac{f(b)-f(a)}{b-a}(x-a)+f(a)$.

Como a função $h(x)$ é polinomial, ela é contínua e derivável em $\mathbb{R}$. 
Tomemos uma função $g(x)=f(x)-h(x)$. Essa função determina a distância vertical entre um ponto $(x, f(x))$ do gráfico de $f$ e o ponto correspondente na reta secante $\stackrel{\leftrightarrow}{P Q}$

Temos, assim:

$$
g(x)=f(x)-\frac{f(b)-f(a)}{b-a}(x-a)-f(a) .
$$

Observemos que a função $g(x)$ cumpre as hipóteses do Teorema de Rolle em $[a, b]$. Com efeito,

(i) $f(x)$ e $h(x)$ são contínuas em $[a, b], \operatorname{logo} g(x)$ é contínua em $[a, b]$.

(ii) $f(x)$ e $h(x)$ são deriváveis em $(a, b), \log o g(x)$ é derivável em $(a, b)$.

(iii) $g(a)=g(b)=0$, pois

$g(a)=f(a)-\frac{f(b)-f(a)}{b-a}(a-a)-f(a)=0$ e $g(b)=f(b)-\frac{f(b)-f(a)}{b-a}(b-a)-f(a)=0$.

Logo, existe $c \in(a, b)$ tal que $g^{\prime}(c)=0$. Como

$g^{\prime}(x)=f^{\prime}(x)-\frac{f(b)-f(a)}{b-a}$, temos $g^{\prime}(c)=f^{\prime}(c)-\frac{f(b)-f(a)}{b-a}=0$ e, assim, segue-se que

$$
f^{\prime}(c)=\frac{f(b)-f(a)}{b-a}
$$

Exemplo: 6.1. Ilustraremos a aplicação do Teorema do Valor Médio em uma função específica.

Solução: 6.1.1. Consideremos a função $f(x)=x^{3}-2 x, a=0$ e $b=2$. Como $f$ é polinomial, temos que ela é contínua e derivável em $\mathbb{R}$; particularmente, $f$ é contínua em $[0,2]$ e derivável em $(0,2)$. Portanto, o Teorema do Valor Médio nos garante que existe $c \in(0,2)$ tal que

$$
f^{\prime}(c)=\frac{f(2)-f(0)}{2-0}
$$

Mas, $f(0)=0, f(2)=4$ e $f^{\prime}(x)=3 x^{2}-2$. Então, temos $3 c^{2}-2=\frac{4-0}{2-0}$, ou seja, $3 c^{2}-2=2$ o que resulta em $c= \pm \frac{2 \sqrt{3}}{3}$.

No entanto, devemos ter $c \in(0,2), \operatorname{logo} c=\frac{2 \sqrt{3}}{3}$. 


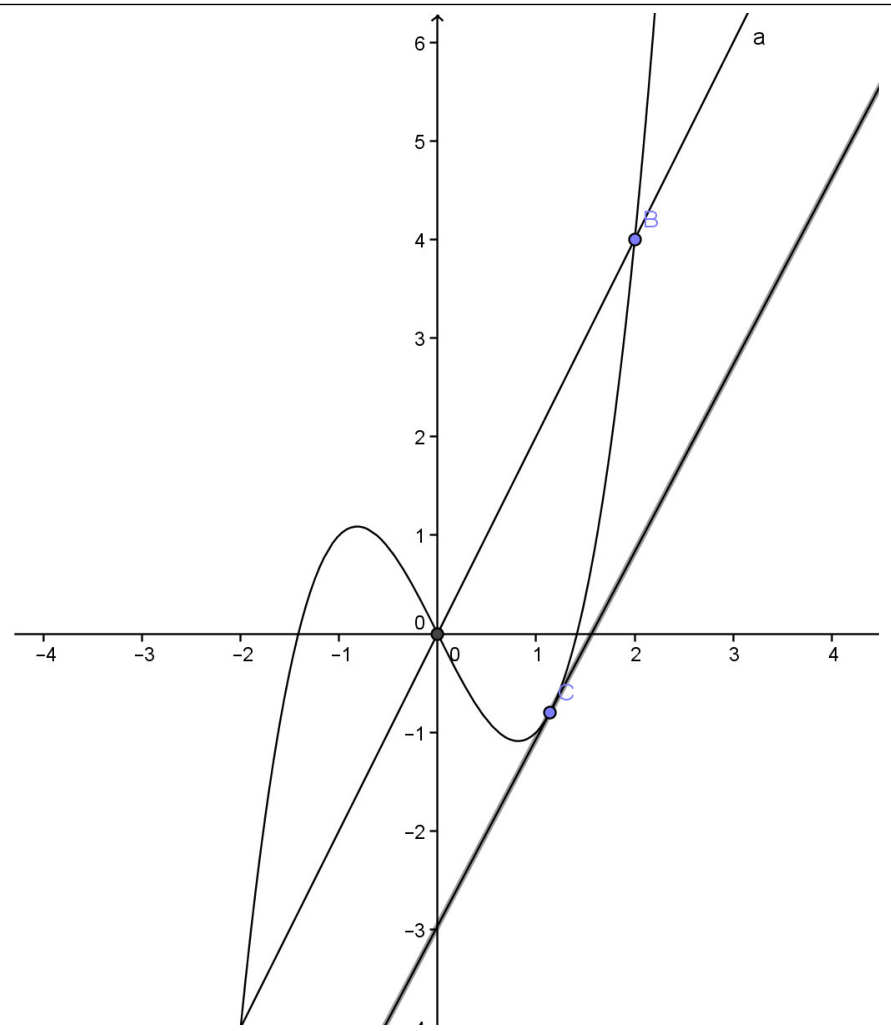

Figura 6.2: Reta tangente em $c=\frac{2 \sqrt{3}}{3}$ paralela à reta secante $O B$

Exemplo: 6.2. Em cada caso, verifique se a função cumpre as condições de aplicação do Teorema do Valor Médio. Caso cumpra, determine o ponto em que isso ocorre.

a)

$$
f(x)=\left\{\begin{array}{cc}
\frac{x-1}{x-2} & \text { se } x \neq 2 \\
1 & \text { se } x=2
\end{array},[1,2]\right.
$$

Solução: 6.1.2. Observemos que

$$
\lim _{x \rightarrow 2^{-}} f(x)=\lim _{x \rightarrow 2^{-}} \frac{x-1}{x-2}=-\infty \neq \infty=\lim _{x \rightarrow 2^{+}} \frac{x-1}{x-2}=\lim _{x \rightarrow 2^{+}} f(x) .
$$

Logo, não existe $\lim _{x \rightarrow 2} f(x)$ e, portanto, $f$ não é contínua em $x=2$, de onde se conclui que $f$ não é contínua em $[1,2]$, não cumprindo, assim, as condições de aplicação do Teorema do Valor Médio.

b)

$$
g(x)=\left\{\begin{array}{ll}
x^{2} & \text { se } x \leq 1 \\
2 x & \text { se } x>1
\end{array},[1,2]\right.
$$

- Para $x<1, g^{\prime}(x)=\left(x^{2}\right)^{\prime}=2 x$;

- Para $x>1, g^{\prime}(x)=(2 x)^{\prime}=2$; 
- Para $x=1$,

i.

$$
\begin{aligned}
g_{-}^{\prime}(1) & =\lim _{h \rightarrow 0^{-}} \frac{f(1+h)-f(1)}{h} \\
& =\lim _{h \rightarrow 0^{-}} \frac{(1+h)^{2}-1^{2}}{h} \\
& =\lim _{h \rightarrow 0^{-}} \frac{1+2 h+h^{2}-1}{h} \\
& =\lim _{h \rightarrow 0^{-}} \frac{h(2+h)}{h} \\
& =\lim _{h \rightarrow 0^{-}}(2+h) \\
& =2 ;
\end{aligned}
$$

ii.

$$
\begin{aligned}
g_{+}^{\prime}(1) & =\lim _{h \rightarrow 0^{+}} \frac{f(1+h)-f(1)}{h} \\
& =\lim _{h \rightarrow 0^{+}} \frac{2(1+h)-2 \cdot 1}{h} \\
& =\lim _{h \rightarrow 0^{+}} \frac{2+2 h-2}{h} \\
& =\lim _{h \rightarrow 0^{+}} \frac{2 h}{h} \\
& =\lim _{h \rightarrow 0^{+}} 2 \\
& =2 ;
\end{aligned}
$$

iii. $g^{\prime}(1)=2 \cdot 1=2$;

iv. $g_{-}^{\prime}(1)=g_{+}^{\prime}(1)=g^{\prime}(1)=2$.

Logo, $g$ é contínua e derivável em $\mathbb{R}$.

Em particular, $g$ é contínua em $[0,2]$ e derivável em $(0,2)$. Então, pelo Teorema do Valor Médio, existe $c \in(0,2)$ tal que

$$
g^{\prime}(c)=\frac{g(2)-g(0)}{2-0}=\frac{2 \cdot 2-0^{2}}{2-0}=2 .
$$

Agora, basta determinarmos os valores de $x$ para os quais temos $g^{\prime}(x)=2$. Assim, temos

(1) para todo $x \leq 1, g^{\prime}(x)=2$, ou seja, $2 x=2$, então $x=1$.

(2) para todo $x>1, g^{\prime}(x)=2$, então $2=2$.

Logo, por (1) e (2), concluímos que $c=1$. 
Exemplo: 6.3. Seja uma função $f: \mathbb{R} \rightarrow \mathbb{R}$ contínua e derivável em $\mathbb{R}$. Suponhamos que $f(0)=-1$ e $f^{\prime}(x) \leq 3$, para todo $x \in \mathbb{R}$. Qual o maior valor que $f(3)$ pode assumir?

Solução: 6.1.3. Como $f$ é contínua e derivável em $\mathbb{R}$, em particular, temos $f$ contínua em $[0,3]$ e derivável em $(0,3)$. Então, pelo Teorema do Valor Médio, existe $c \in(0,3)$ tal que

$f^{\prime}(c)=\frac{f(3)-f(0)}{3-0}$, ou seja, $f^{\prime}(c)=\frac{f(3)+1}{3}$ ou ainda, $f(3)=3 f^{\prime}(c)-1$.

Como $f^{\prime}(x) \leq 3$, para todo $x \in \mathbb{R}$, tem-se que $f^{\prime}(c) \leq 3$. Multiplicando ambos os lados dessa igualdade por 3 , temos $3 f^{\prime}(c) \leq 9$, logo $f(3)=3 f^{\prime}(c)-1 \leq 9-1=8$.

Portanto, o maior valor que $f(3)$ pode assumir é 8.

Existem muitos outros tipos de teoremas de valor médio que são menos conhecidos. Vejamos, por exemplo o teorema de Flett.

Teorema 6.1.2 (Teorema de Flett). Sejam $A$ um subconjunto não vazio de $\mathbb{R}$ e uma função $f: A \rightarrow \mathbb{R}$ contínua em $[a, b]$ e derivável em $(a, b)$, com $f^{\prime}(a)=f^{\prime}(b)$. Então, existe um ponto $c \in(a, b)$ tal que $f(c)-f(a)=(c-a) f^{\prime}(c)$.

A interpretação geométrica desse teorema é que, Se a curva $y=f(x)$ tem uma tangente em cada ponto em $a, b)$, e se as tangentes em $(a, f(a))$ e $(b, f(b))$ são paralelas, então há um ponto intermediário $c$ tal que a tangente em $(c, f(c))$ passe pelo ponto $(a, f(a))$.

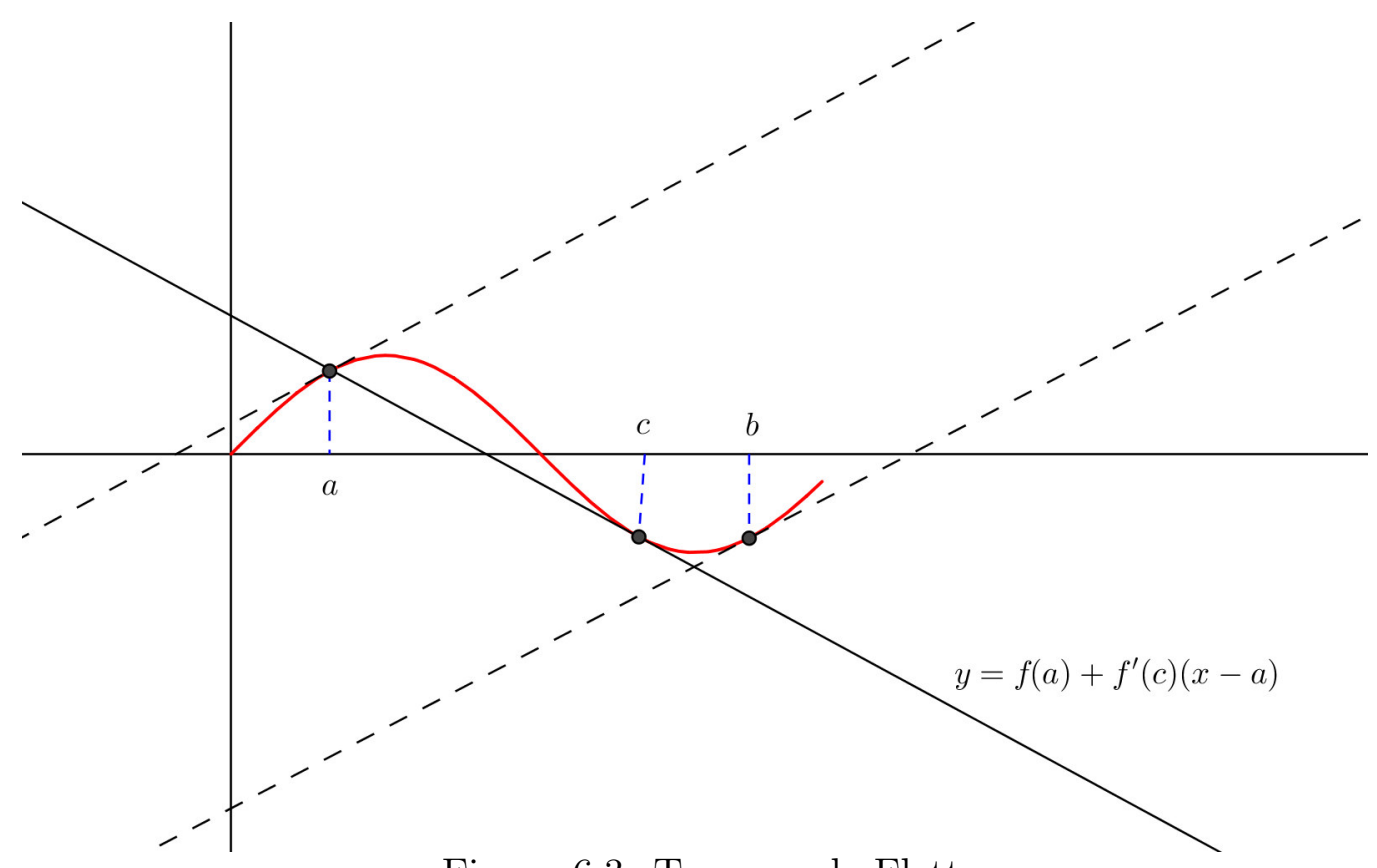

Figura 6.3: Teorema de Flett

Podemos estender o Teorema de Flett para funções reais, a um resultado que não depende da hipótese $f^{\prime}(a)=f^{\prime}(b)$, enunciando o seguinte resultado, a partir do teorema de Flett.

Teorema 6.1.3. Sejam $A$ um subconjunto não vazio de $\mathbb{R}$ e uma função $f: A \rightarrow \mathbb{R}$ contínua em $[a, b]$ e derivável em $(a, b)$, com $f^{\prime}(a)=f^{\prime}(b)$. Então, existe um ponto $c \in(a, b)$ tal que

$$
f(c)=(c-a) f^{\prime}(c)-\frac{1}{2} \frac{f^{\prime}(b)-f^{\prime}(a)}{b-a}(c-a)^{2}+f(a) .
$$


Demonstração. Consideremos a função auxiliar $h:[a, b] \rightarrow \mathbb{R}$ definida por

$h(x)=f(x)-\frac{1}{2} \frac{f^{\prime}(b)-f^{\prime}(a)}{b-a}(x-a)^{2}$.

Temos que $h$ é contínua em $[a, b]$ e derivável em $(a, b)$, e

$h^{\prime}(x)=f^{\prime}(x)-\frac{f^{\prime}(b)-f^{\prime}(a)}{b-a}(x-a)$. Daí,

- (i) $h^{\prime}(a)=f^{\prime}(a)-\frac{f^{\prime}(b)-f^{\prime}(a)}{b-a}(a-a)=f^{\prime}(a)$.

- $\left(\right.$ ii) $h^{\prime}(b)=f^{\prime}(b)-\frac{f^{\prime}(b)-f^{\prime}(a)}{b-a}(b-a)=f^{\prime}(a)$.

De $(i)$ e $(i i)$ temos que $h^{\prime}(a)=h^{\prime}(b)=f^{\prime}(a)$, condição que associada ao fato de $h$ ser contínua em $[a, b]$ e derivável em $(a, b)$ nos permite aplicar o Teorema de Flett em $h$. Daí, obtemos $h(c)-h(a)=(c-a) f^{\prime}(c)$, para algum $c \in(a, b)$. Reescrevendo $h$ e $h^{\prime}$ em termos de $f$ obtemos o resultado esperado.

Exemplo: 6.4. Ilustraremos a aplicação do Teorema 6.1.3 em uma função específica.

Solução: 6.1.4. Consideremos a função $f(x)=x^{2}-1, a=-1$ e $b=1$. Como $f$ é polinomial, temos que ela é contínua e derivável em $\mathbb{R}$; particularmente, $f$ é contínua em $[-1,1]$ e derivável em $(-1,1)$. Portanto, o Teorema 6.1.3 nos garante que existe $c \in(-1,1)$ tal que

$$
\begin{gathered}
f(c)-f(-1)=(c-(-1)) f^{\prime}(c)-\frac{1}{2} \frac{f^{\prime}(1)-f^{\prime}(-1)}{1-(-1)}(c-(-1))^{2}, \text { e assim, } \\
f(c)=(c+1)\left[f^{\prime}(c)-(c+1)\right] .
\end{gathered}
$$

De fato, tomando-se uma função auxiliar

$h:[a, b] \rightarrow \mathbb{R}$ definida por

$h(x)=f(x)-\frac{1}{2} \frac{f^{\prime}(b)-f^{\prime}(a)}{b-a}(x-a)^{2}$, substituindo $f(x), a$ e $b$, teremos

$$
h(x)=\left(x^{2}-1\right)-(x+1)^{2} .
$$

Temos que $h$ é contínua em $[a, b]$ e derivável em $(a, b)$, e $h^{\prime}(x)=2 x-2(x+1)$. Daí,

- $h^{\prime}(-1)=2(-1)-2(-1+1)=-2$.

- $h^{\prime}(1)=2 \cdot 1-2(1+1)=-2$.

De $(i)$ e $(i i)$ temos que $h^{\prime}(-1)=h^{\prime}(1)=f^{\prime}(-1)$, pois $h^{\prime}(x)=2 x$, logo $f^{\prime}(-1)=2(-1)=-2$. Portanto, a função $h$ satisfaz as três condições de aplicação do Teorema 6.1.3, que ao ser aplicado resulta em

$h(c)-h(1)=(c-1) f^{\prime}(c)$, ou seja, $\left[\left(c^{2}-1\right)-(c+1)^{2}\right]-\left[\left((-1)^{2}-1\right)-(-1+1)^{2}\right]=(c+1) \cdot 2 c$, o que resulta em $c=1$. 


\section{UMA APLICAÇÃO PARTICULAR DO TEOREMA DO VALOR MÉDIO}

\subsection{Uma Aplicação Particular do Teorema do Valor Médio}

Partindo-se do capítulo anterior temos que, se uma função $f$ é contínua em $[a, b]$ e derivável em $(a, b)$, o Teorema do Valor Médio afirma que existe um número $c \in(a, b)$ tal que

$f^{\prime}(c)=\frac{f(b)-f(a)}{b-a}$,

o que, geometricamente, estabelece que, se uma função $y=f(x)$ é contínua em $[a, b]$ e derivável em $(a, b)$, então existe pelo menos um ponto $c$ entre os pontos $a$ e $b$ onde a reta tangente à curva é paralela à reta suporte da corda que une dois pontos quaisquer $P(a, f(a))$ e $Q(b, f(b))$. Como exemplo, tomemos a função $f(x)=x^{2}-9$ no intervalo $[-3,3]$. Então, por ser polinomial temos que $f$ é contínua em $[-3,3]$, derivável em $(-3,3)$. O Teorema do Valor Médio nos assegura que deve existir $c \in(-3,3)$ tal que

$f^{\prime}(c)=\frac{f(b)-f(a)}{b-a}=\frac{f(3)-f(-3)}{3-(-3)}=\left(\frac{\left(3^{2}-9\right)-\left[(-3)^{2}-9\right]}{3+3}=0\right.$.

Como $f^{\prime}(x)=2 x$, temos $f^{\prime}(x)=0 \Rightarrow 2 x=0 \Rightarrow x=0$ e, portanto, vemos que $c=0$.

Agora, atentemos para o seguinte caso particular enunciado no teorema a seguir.

Teorema 7.1.1. Teorema Seja $f$ uma função contínua em $[a, b]$ e derivável em $(a, b)$, $A(a, f(a))$ e $B(b, f(b))$ dois pontos do gráfico de $f$ e $h(x)$ a função que define a reta $\stackrel{\leftrightarrow}{A B}$ secante ao gráfico de $f$. Seja $g(x)=f(x)-h(x)$ a função que determina a distância vertical entre um ponto $(x, f(x))$ do gráfico de $f$ e o ponto correspondente na reta secante $\stackrel{\leftrightarrow}{A B}$. Então, dado $k$ real existe um número $c \in(a, b)$ tal que:

$f^{\prime}(c)=\frac{f(b)-f(a)}{b-a}+k g(c)$.

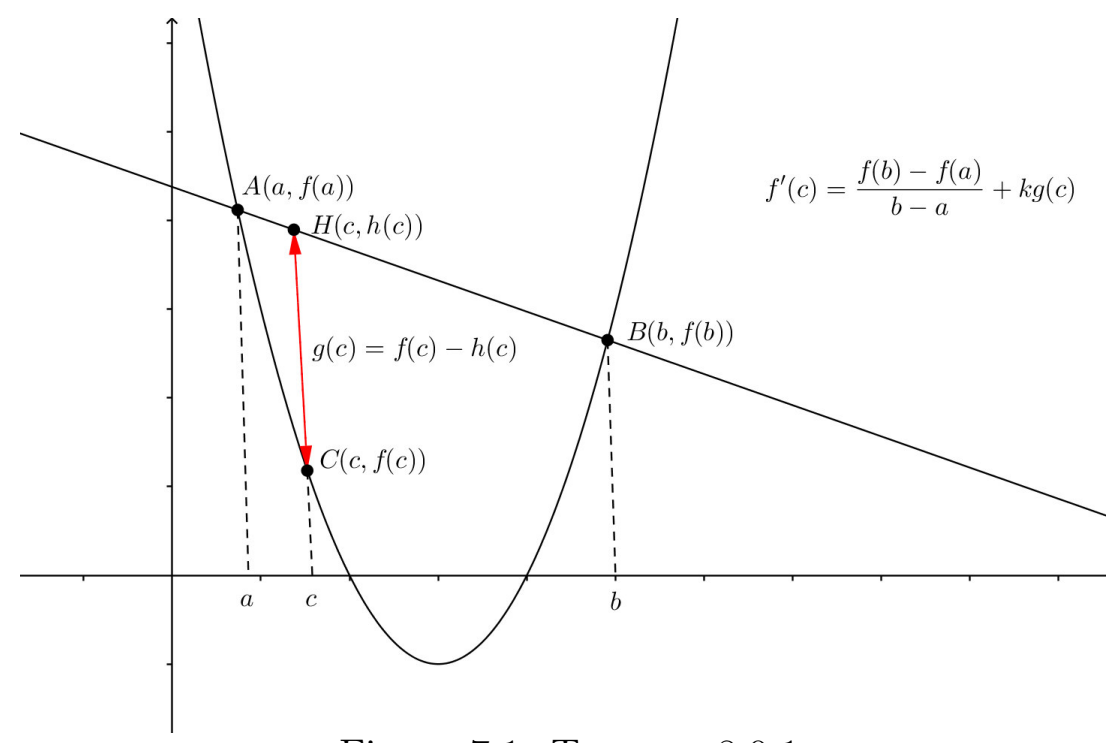

Figura 7.1: Teorema 8.0.1 
Observação: 7.1.1. Como uma aplicação particular do teorema do Valor Médio, este teorema está relacionado à inclinação da reta tangente à uma curva qualquer, observandose assim que uma das condições necessárias é que a função que define a curva seja diferenciável apenas no intervalo aberto $(a, b)$, o que equivale a afirmar que a função tem que ser diferenciável apenas nos pontos interiores do intervalo $[a, b]$, ou seja, diferenciável em $\operatorname{int}[a, b]$.

Demonstração. Sejam uma curva $y=f(x)$ e uma reta secante à essa curva nos pontos $P(a, f(a))$ e $Q(b, f(b))$. A equação da reta $P Q$, conforme o capítulo anterior, é

$y-f(a)=\frac{f(b)-f(a)}{b-a}(x-a)$

e, fazendo $y=h(x)$, teremos:

$h(x)=\frac{f(b)-f(a)}{b-a}(x-a)+f(a)$,

que é uma função polinomial e, portanto, contínua e derivável em $\mathbb{R}$.

Tomemos uma função $g(x)=f(x)-h(x)$. Essa função determina a distância vertical entre um ponto $(x, f(x))$ do gráfico de $f$ e o ponto correspondente na reta secante $\overleftrightarrow{P Q}$. Temos, assim:

$g(x)=f(x)-\frac{f(b)-f(a)}{b-a}(x-a)-f(a)$.

Observemos que a função $g(x)$ cumpre as hipóteses do Teorema de Rolle. Com efeito,

(i) $f(x)$ e $h(x)$ são contínuas em $[a, b], \operatorname{logo} g(x)$ é contínua em $[a, b]$.

(ii) $f(x)$ e $h(x)$ são deriváveis em $(a, b), \log o g(x)$ é derivável em $(a, b)$.

(iii) $g(a)=g(b)=0$, pois

$g(a)=f(a)-\frac{f(b)-f(a)}{b-a}(a-a)-f(a)=0$

e

$g(b)=f(b)-\frac{f(b)-f(a)}{b-a}(b-a)-f(a)=0$.

Portanto, pelo Teorema 6.1.1, existe um ponto $c \in(a, b)$ tal que $g^{\prime}(c)=k g(c)$.

Como

$g^{\prime}(x)=f^{\prime}(x)-\frac{f(b)-f(a)}{b-a}$,

temos:

$g^{\prime}(c)=f^{\prime}(c)-\frac{f(b)-f(a)}{b-a}=k g(c)$

e, desta forma

$f^{\prime}(c)=\frac{f(b)-f(a)}{b-a}+k g(c)$,

o que, geometricamente, quer dizer que existe um ponto $c$ entre os pontos $a$ e $b$ onde a reta tangente à curva possui inclinação igual ao coeficiente angular da reta suporte da corda que une os pontos $P(a, f(a))$ e $Q(b, f(b))$ adicionado a um múltiplo da distância vertical entre o ponto $(c, f(c))$ do gráfico de $f$ e o ponto correspondente na reta secante $\stackrel{\leftrightarrow}{P Q}$ 
Exemplo: 7.1. Determine $k \in \mathbb{R}$ em função de um ponto $c$ qualquer entre os pontos -1 e 1 onde a reta tangente à curva $f(x)=x^{2}-2 x-1$ possui inclinação igual ao coeficiente angular da reta suporte da corda que une os pontos $P(-1, f(-1))$ e $Q(1, f(1))$ adicionado a um múltiplo de $k$ da distância vertical entre o ponto $(c, f(c))$ do gráfico de $f$ e o ponto correspondente na reta secante $\stackrel{\leftrightarrow}{P Q}$.

Solução: 7.1.1. Por ser polinomial, temos que $f$ é contínua e derivável em $\mathbb{R}$; em particular, $f$ é contínua em $[-1,1]$ e derivável em $(-1,1)$. Sabendo-se que $f(-1)=2$ e $f(1)=-2$, a equação da reta secante à essa curva nos pontos $P(-1, f(-1))$ e $Q(1, f(1))$ é

$y-f(-1)=\frac{f(1)-f(-1)}{1-(-1)}(x-(-1)) \Rightarrow y-2=\frac{-2-2}{2}(x+1) \Rightarrow y-2=-2 x-2 \Rightarrow y=-2 x$

e, fazendo $y=h(x)$, teremos:

$h(x)=-2 x$,

que é uma função polinomial e, portanto, contínua e derivável em $\mathbb{R}$.

Tomemos uma função $g(x)=f(x)-h(x)$. Essa função determina a distância vertical entre um ponto $(x, f(x))$ do gráfico de $f$ e o ponto correspondente na reta secante $\stackrel{\leftrightarrow}{P Q}$

Temos, assim:

$g(x)=f(x)-h(x) \Rightarrow g(x)=\left(x^{2}-2 x-1\right)-(-2 x) \Rightarrow g(x)=x^{2}-1$.

Observemos que a função $g(x)$ cumpre as hipóteses do Teorema de Rolle. Com efeito,

(i) $f(x)$ e $h(x)$ são contínuas em $[-1,1], \operatorname{logo} g(x)$ é contínua em $[-1,1]$.

(ii) $f(x)$ e $h(x)$ são deriváveis em $(-1,1)$, logo $g(x)$ é derivável em $(-1,1)$.

(iii) $g(-1)=g(1)=0$, pois

$g(-1)=(-1)^{2}-1=0$

$\mathrm{e}$

$g(1)=1^{2}-1=0$

Portanto, existe um ponto $c \in(-1,1)$ tal que

$f^{\prime}(c)=\frac{f(b)-f(a)}{b-a}+k g(c) \Rightarrow f^{\prime}(c)=\frac{f(1)-f(-1)}{1-(-1)}+k g(c) \Rightarrow 2 c-2=\frac{-2-2}{1-(-1)}+k\left(c^{2}-1\right)$

$\Rightarrow 2 c-2=-2+k\left(c^{2}-1\right) \Rightarrow k=\frac{2 c}{c^{2}-1}$.

Exemplo: 7.2. Determine o ponto $c$ entre os pontos 0 e 2 onde a reta tangente à curva $f(x)=x^{2}-4 x+3$ possui inclinação igual ao coeficiente angular da reta suporte da corda que une os pontos $P(0, f(0))$ e $Q(2, f(2))$ adicionado ao triplo da distância vertical entre o ponto $(c, f(c))$ do gráfico de $f$ e o ponto correspondente na reta secante $\overleftrightarrow{P Q}$.

Solução: 7.1.2. Por ser polinomial, temos que $f$ é contínua e derivável em $\mathbb{R}$; em particular, $f$ é contínua em $[0,2]$ e derivável em $(0,2)$. Sabendo-se que $f(0)=3$ e $f(2)=-1$, a equação da reta secante à essa curva nos pontos $P(0, f(0))$ e $Q(2, f(2))$ é

$y-f(0)=\frac{f(2)-f(0)}{2-0}(x-0) \Rightarrow y-3=\frac{-1-3}{2} x \Rightarrow y-3=-2 x$.

e, fazendo $y=h(x)$, teremos:

$h(x)-3=-2 x \Rightarrow h(x)=-2 x+3$, 
que é uma função polinomial e, portanto, contínua e derivável em $\mathbb{R}$.

Tomemos uma função $g(x)=f(x)-h(x)$. Essa função determina a distância vertical entre um ponto $(x, f(x))$ do gráfico de $f$ e o ponto correspondente na reta secante $\stackrel{\leftrightarrow}{P Q}$

Temos, assim:

$g(x)=f(x)-h(x) \Rightarrow g(x)=\left(x^{2}-4 x+3\right)-(-2 x+3) \Rightarrow g(x)=x^{2}-2 x$.

efeito,

Observemos que a função $g(x)$ cumpre as hipóteses do Teorema de Rolle. Com

(i) $f(x)$ e $h(x)$ são contínuas em $[0,2]$, logo $g(x)$ é contínua em $[0,2]$.

(ii) $f(x)$ e $h(x)$ são deriváveis em $(0,2)$, logo $g(x)$ é derivável em $(0,2)$.

(iii) $g(0)=g(2)=0$, pois

$g(0)=0^{2}-2 \cdot 0=0$

e

$g(2)=2^{2}-2 \cdot 2=0$.

Portanto, existe um ponto $c \in(0,2)$ tal que

$f^{\prime}(c)=\frac{f(b)-f(a)}{b-a}+k g(c) \Rightarrow 2 c-4=\frac{-1-3}{2-0}+3\left(c^{2}-2 c\right) \Rightarrow 2 c-4=-2+3 c^{2}-6 c$

$\Rightarrow 3 c^{2}-8 c+2=0 \Rightarrow c=\frac{4 \pm \sqrt{10}}{3}$.

No entanto, devemos ter $c \in(0,2)$; logo,

$c=\frac{4-\sqrt{10}}{3}$.

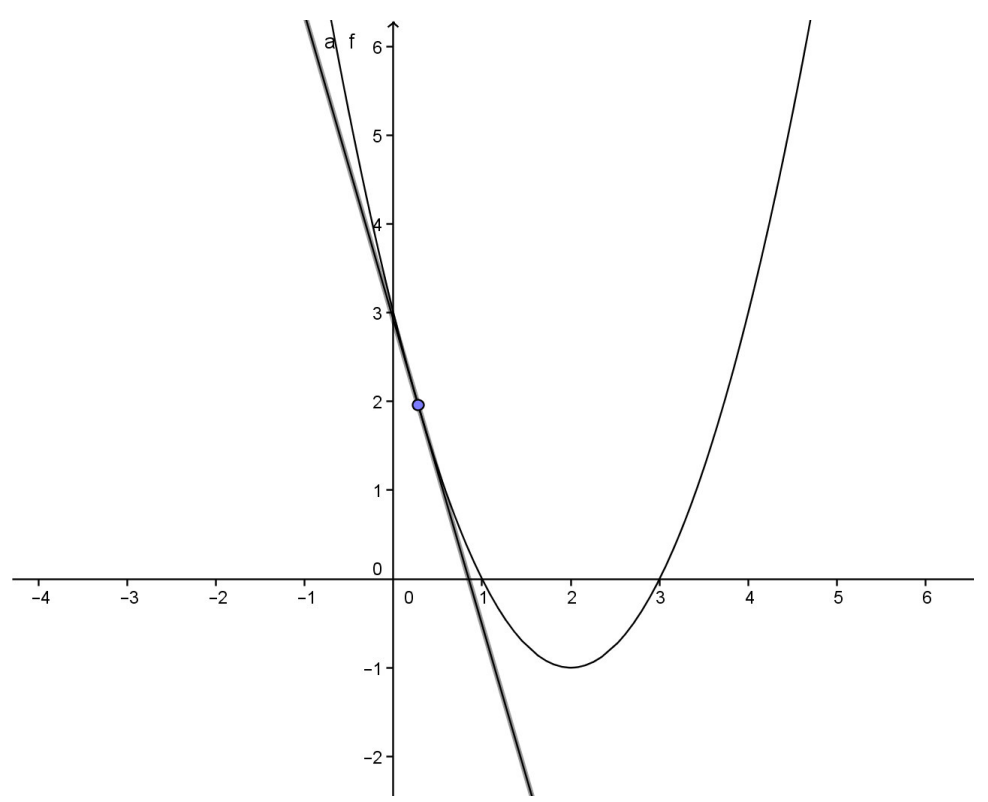

Figura 7.2: Reta tangente à curva $y=x^{2}-4 x+3$ no ponto $c=\frac{4-\sqrt{10}}{3}$ 
Exemplo: 7.3. Encontre o ponto $c$ entre os pontos 1 e 3 onde a reta tangente à curva $f(x)=-x^{2}+3 x-2$ possui inclinação igual ao coeficiente angular da reta suporte da corda que une os pontos $P(1, f(1))$ e $Q(3, f(3))$ adicionado ao dobro da distância vertical entre o ponto $(c, f(c))$ do gráfico de $f$ e o ponto correspondente na reta secante $\overleftrightarrow{P Q}$.

Solução: 7.1.3. Solução: Por ser polinomial, temos que $f$ é contínua e derivável em $\mathbb{R}$; em particular, $f$ é contínua em $[1,3]$ e derivável em $(1,3)$. Sabendo-se que $f(1)=0$ e $f(3)=-2$, a equação da reta secante à essa curva nos pontos $P(1, f(1))$ e $Q(3, f(3))$ é

$y-f(1)=\frac{f(3)-f(1)}{3-1}(x-1) \Rightarrow y-0=\frac{-2-0}{3-1}(x-1) \Rightarrow y=-x+1$.

e, fazendo $y=h(x)$, teremos:

$h(x)=-x+1$,

que é uma função polinomial e, portanto, contínua e derivável em $\mathbb{R}$.

Tomemos uma função $g(x)=f(x)-h(x)$. Essa função determina a distância vertical entre um ponto $(x, f(x))$ do gráfico de $f$ e o ponto correspondente na reta secante $\overleftrightarrow{P Q}$

Temos, assim:

$g(x)=f(x)-h(x) \Rightarrow g(x)=\left(-x^{2}+3 x-2\right)-(-x+1) \Rightarrow g(x)=-x^{2}+4 x-3$.

efeito,

Observemos que a função $g(x)$ cumpre as hipóteses do Teorema de Rolle. Com

(i) $f(x)$ e $h(x)$ são contínuas em $[1,3]$, logo $g(x)$ é contínua em $[1,3]$.

(ii) $f(x)$ e $h(x)$ são deriváveis em $(1,3)$, logo $g(x)$ é derivável em $(1,3)$.

(iii) $g(1)=g(3)=0$, pois

$g(1)=-1^{2}+4 \cdot 1-3=0$ e $g(3)=-3^{2}+4 \cdot 3-3=0$.

Portanto, existe um ponto $c \in(1,3)$ tal que

$$
\begin{aligned}
f^{\prime}(c)=\frac{f(b)-f(a)}{b-a}+k g(c) & \Rightarrow-2 c+3=\frac{-2-0}{3-1}+2\left(-c^{2}+4 c-3\right) \\
\Rightarrow-2 c+3 & =-1-2 c^{2}+8 c-6 \Rightarrow c^{2}-5 c+5=0 \Rightarrow c=\frac{5 \pm \sqrt{5}}{2} .
\end{aligned}
$$

Porém, devemos ter $c \in(1,3) ; \operatorname{logo}, c=\frac{5-\sqrt{5}}{2}$.

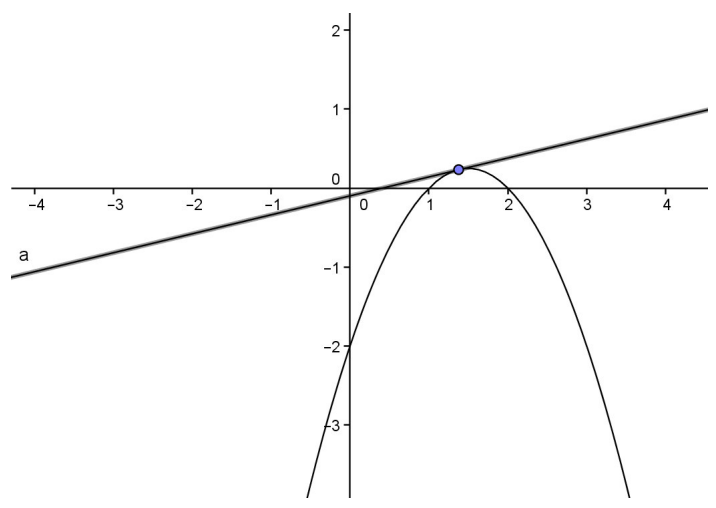

Figura 7.3: Reta tangente à curva $y=-x^{2}+3 x-2$ no ponto $c=\frac{5-\sqrt{5}}{2}$ 
Exemplo: 7.4. Seja a curva definida por $f(x)=x^{2}-x$. Determine o ponto $c$ entre os pontos -2 e 2 onde a reta tangente à esta curva possui inclinação igual ao coeficiente angular da reta suporte da corda que une os pontos $P(-2, f(-2))$ e $Q(2, f(2))$ adicionado à terça parte da distância vertical entre o ponto $(c, f(c))$ do gráfico de $f$ e o ponto correspondente na reta secante $\overleftrightarrow{P Q}$.

Solução: 7.1.4. Como $f$ é polinomial, é contínua e derivável em $\mathbb{R}$; em particular, $f$ é contínua em $[-2,2]$ e derivável em $(-2,2)$. Sabendo-se que $f(-2)=6$ e $f(2)=2$, a equação da reta secante à essa curva nos pontos $P(-2, f(-2))$ e $Q(2, f(2))$ é

$y-f(-2)=\frac{f(2)-f(-2)}{2-(-2)}(x-(-2)) \Rightarrow y-6=\frac{2-6}{2+2}(x+2) \Rightarrow y-6=-x-2$.

e, fazendo $y=h(x)$, teremos:

$h(x)-6=-x-2 \Rightarrow h(x)=-x+4$,

que é uma função polinomial e, portanto, contínua e derivável em $\mathbb{R}$.

Tomemos uma função $g(x)=f(x)-h(x)$, que determina a distância vertical entre um ponto $(x, f(x))$ do gráfico de $f$ e o ponto correspondente na reta secante $\stackrel{\leftrightarrow}{P Q}$.

Temos, assim:

$g(x)=f(x)-h(x) \Rightarrow g(x)=\left(x^{2}-x\right)-(-x+4) \Rightarrow g(x)=x^{2}-4$.

Observemos que a função $g(x)$ cumpre as hipóteses do Teorema de Rolle. Com efeito,

(i) $f(x)$ e $h(x)$ são contínuas em $[-2,2]$, logo $g(x)$ é contínua em $[-2,2]$.

(ii) $f(x)$ e $h(x)$ são deriváveis em $(-2,2)$, logo $g(x)$ é derivável em $(-2,2)$.

(iii) $g(-2)=g(2)=0$, pois

$g(-2)=(-2)^{2}-4=0$ e $g(2)=2^{2}-4=0$.

Portanto, existe um ponto $c \in(-2,2)$ tal que

$f^{\prime}(c)=\frac{f(b)-f(a)}{b-a}+k g(c) \Rightarrow 2 c-1=\frac{2-6}{2-(-2)}+\frac{1}{3}\left(c^{2}-4\right)$

$\Rightarrow 2 c-1=-1+\frac{c^{2}}{3}-\frac{4}{3} \Rightarrow c^{2}-6 c-4=0 \Rightarrow c=3 \pm \sqrt{13}$.

Entretanto, devemos ter $c \in(-2,2)$; $\operatorname{logo}, c=3-\sqrt{13}$.

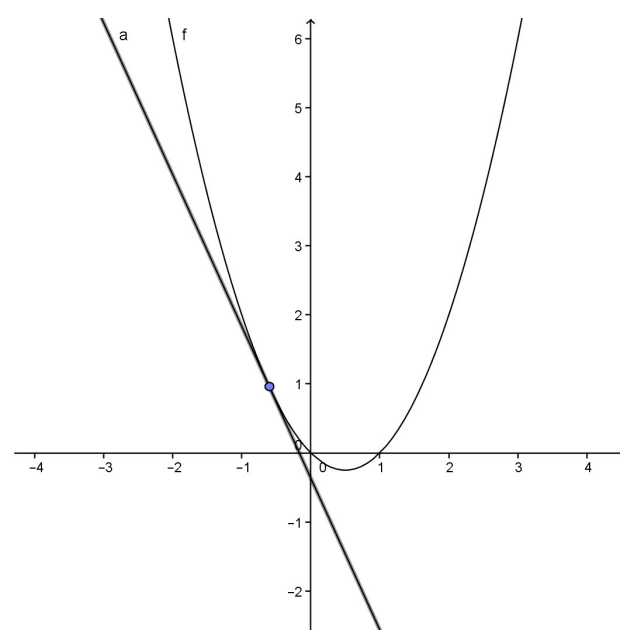

Figura 7.4: Reta tangente à curva $y=x^{2}-x$ no ponto $c=3-\sqrt{13}$ 


\section{APLICAÇÃO DO TEOREMA 8.1.1 NO ENSINO MÉDIO}

\subsection{Aplicação do Teorema 8.1.1 no Ensino Médio}

Como se pode observar no capítulo anterior, o Teorema 8.1.1 aborda conceitos da Geometria Analítica, como coeficiente angular, equação da reta tangente e distância entre dois pontos.

Desta forma, o Teorema 8.1.1 pode ser aplicado no Ensino Médio, nas turmas de terceiro ano, na solução de alguns exercícios específicos que envolvem tais conceitos de Geometria Analítica.

Vejamos a seguir alguns exemplos de exercícios que podem ser aplicados nas turmas de terceiro ano do Ensino Médio, onde se aplica o Teorema 8.1.1.

Exemplo: 8.1. Determine o ponto $c$ entre os pontos -1 e 1 onde a reta tangente à curva $f(x)=x^{2}-2 x+1$ possui inclinação igual ao coeficiente angular da reta suporte da corda que une os pontos $P(-1, f(-1))$ e $Q(1, f(1))$ adicionado ao quádruplo da distância vertical entre o ponto $(c, f(c))$ do gráfico de $f$ e o ponto correspondente na reta secante $\stackrel{\leftrightarrow}{P Q}$

Solução: 8.1.1. Por ser polinomial, temos que $f$ é contínua e derivável em $\mathbb{R}$; em particular, $f$ é contínua em $[-1,1]$ e derivável em $(-1,1)$. Sabendo-se que $f(-1)=4$ e $f(1)=0$, a equação da reta secante à essa curva nos pontos $P(-1, f(-1))$ e $Q(1, f(1))$ é $y-f(1)=\frac{f(1)-f(-1)}{1-(-1)}(x-1) \Rightarrow y-0=\frac{0-4}{1+1}(x-1) \Rightarrow y=-2(x-1) \Rightarrow y=-2 x+2$.

e, fazendo $y=h(x)$, teremos:

$h(x)=-2 x+2$,

que é uma função polinomial e, portanto, contínua e derivável em $\mathbb{R}$.

Tomemos uma função $g(x)=f(x)-h(x)$. Essa função determina a distância vertical entre um ponto $(x, f(x))$ do gráfico de $f$ e o ponto correspondente na reta secante $\stackrel{\leftrightarrow}{P Q}$

Temos, assim:

$g(x)=f(x)-h(x) \Rightarrow g(x)=\left(x^{2}-2 x+1\right)-(-2 x+2) \Rightarrow g(x)=x^{2}-2 x+1+2 x-2$ $\Rightarrow g(x)=x^{2}-1$.

efeito,

Observemos que a função $g(x)$ cumpre as hipóteses do Teorema de Rolle. Com

(i) $f(x)$ e $h(x)$ são contínuas em $[-1,1]$, logo $g(x)$ é contínua em $[-1,1]$.

(ii) $f(x)$ e $h(x)$ são deriváveis em $(-1,1)$, logo $g(x)$ é derivável em $(-1,1)$.

(iii) $g(-1)=g(1)=0$, pois

$g(-1)=(-1)^{2}-1=0$ e $g(1)=1^{2}-1=0$.

Portanto, existe um ponto $c \in(-1,1)$ tal que 


$$
\begin{aligned}
& f^{\prime}(c)=\frac{f(1)-f(-1)}{1-(-1)}+4 g(c) \Rightarrow 2 c-2=\frac{0-4}{1+1}+4\left(c^{2}-1\right) \Rightarrow 2 c-2=-2+4 c^{2}-4 \\
& \Rightarrow 4 c^{2}-2 c-4=0 \Rightarrow c=\frac{1 \pm \sqrt{17}}{4} .
\end{aligned}
$$

No entanto, devemos ter $c \in(-1,1) ; \operatorname{logo}, c=\frac{1-\sqrt{17}}{4}$.

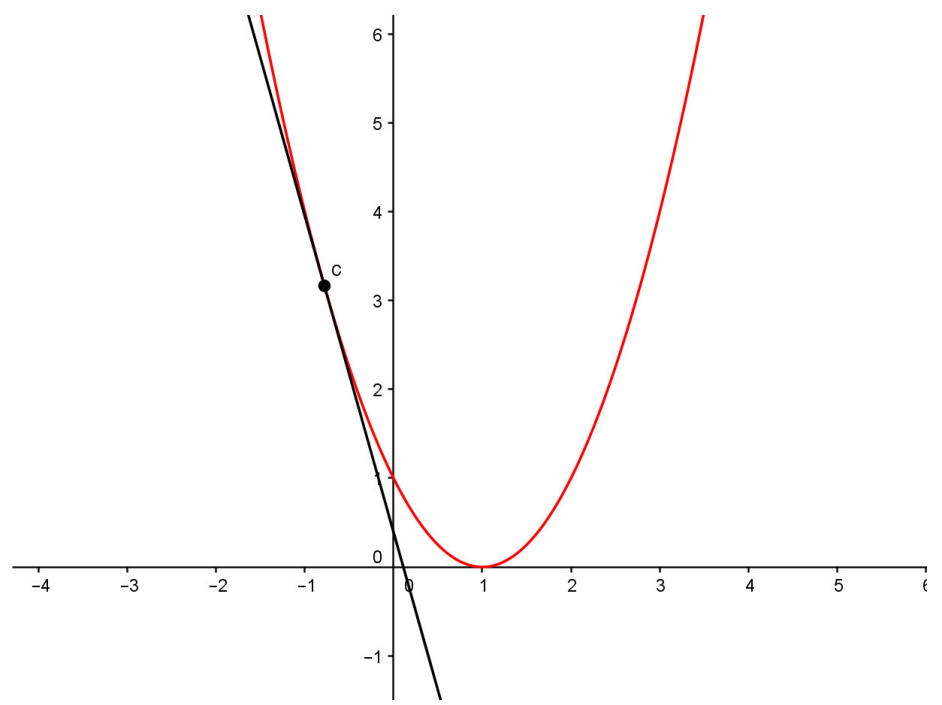

Figura 8.1: Reta tangente à curva $y=x^{2}-2 x+1$ no ponto $c=\frac{1-\sqrt{17}}{4}$

Exemplo: 8.2. Determine o ponto $c$ entre os pontos -1 e 1 onde a reta tangente à curva $f(x)=2 x^{2}-4 x$ possui inclinação igual ao coeficiente angular da reta suporte da corda que une os pontos $P(-1, f(-1))$ e $Q(1, f(1))$ adicionado ao dobro da distância vertical entre o ponto $(c, f(c))$ do gráfico de $f$ e o ponto correspondente na reta secante $\stackrel{\leftrightarrow}{P Q}$.

Solução: 8.1.2. Por ser polinomial, temos que $f$ é contínua e derivável em $\mathbb{R}$; em particular, $f$ é contínua em $[-1,1]$ e derivável em $(-1,1)$. Sabendo-se que $f(-1)=6$ e $f(1)=-2$, a equação da reta secante à essa curva nos pontos $P(-1, f(-1))$ e $Q(1, f(1))$ é

$y-f(1)=\frac{f(1)-f(-1)}{1-(-1)}(x-1) \Rightarrow y-(-2)=\frac{-2-6}{1+1}(x-1) \Rightarrow y+2=-4(x-1) \Rightarrow y=-4 x+2$.

e, fazendo $y=h(x)$, teremos:

$h(x)=-4 x+2$,

que é uma função polinomial e, portanto, contínua e derivável em $\mathbb{R}$.

Tomemos uma função $g(x)=f(x)-h(x)$. Essa função determina a distância vertical entre um ponto $(x, f(x))$ do gráfico de $f$ e o ponto correspondente na reta secante $\stackrel{\leftrightarrow}{P Q}$

Temos, assim:

$g(x)=f(x)-h(x) \Rightarrow g(x)=\left(2 x^{2}-4 x\right)-(-4 x+2) \Rightarrow g(x)=2 x^{2}-4 x+4 x-2$ $\Rightarrow g(x)=2 x^{2}-2$.

efeito,

Observemos que a função $g(x)$ cumpre as hipóteses do Teorema de Rolle. Com

(i) $f(x)$ e $h(x)$ são contínuas em $[-1,1]$, $\log o g(x)$ é contínua em $[-1,1]$.

(ii) $f(x)$ e $h(x)$ são deriváveis em $(-1,1)$, logo $g(x)$ é derivável em $(-1,1)$. 
(iii) $g(-1)=g(1)=0$, pois

$g(-1)=2 \cdot(-1)^{2}-2=0$ e $g(1)=2 \cdot 1^{2}-2=0$.

Portanto, existe um ponto $c \in(-1,1)$ tal que

$f^{\prime}(c)=\frac{f(1)-f(-1)}{1-(-1)}+2 g(c) \Rightarrow 4 c-4=\frac{-2-6}{1+1}+2\left(2 c^{2}-2\right) \Rightarrow 4 c-4=-4+4 c^{2}-4$

$\Rightarrow 4 c^{2}-4 c-4=0 \Rightarrow c=\frac{1 \pm \sqrt{5}}{2}$.

No entanto, devemos ter $c \in(-1,1) ; \operatorname{logo}, c=\frac{1-\sqrt{5}}{2}$.

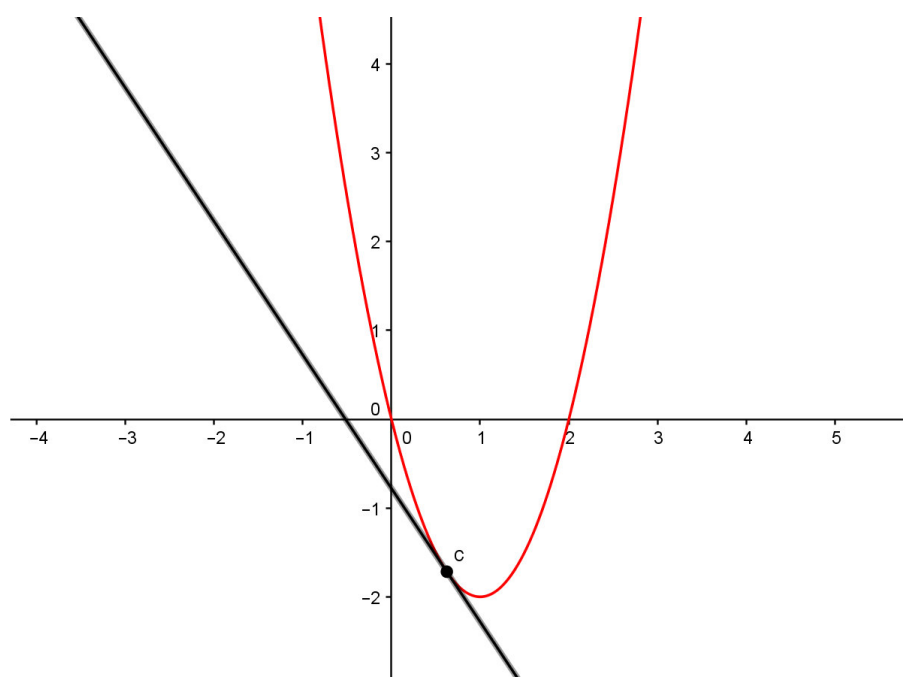

Figura 8.2: Reta tangente à curva $y=2 x^{2}-4 x$ no ponto $c=\frac{1-\sqrt{17}}{4}$

Exemplo: 8.3. Determine o ponto $c$ entre os pontos 0 e 2 onde a reta tangente à curva $f(x)=3 x^{2}-12 x+1$ possui inclinação igual ao coeficiente angular da reta suporte da corda que une os pontos $P(0, f(0))$ e $Q(2, f(2))$ adicionado ao triplo da distância vertical entre o ponto $(c, f(c))$ do gráfico de $f$ e o ponto correspondente na reta secante $\overleftrightarrow{P Q}$.

Solução: 8.1.3. Por ser polinomial, temos que $f$ é contínua e derivável em $\mathbb{R}$; em particular, $f$ é contínua em $[0,2]$ e derivável em $(0,2)$. Sabendo-se que $f(0)=1$ e $f(2)=-11$, a equação da reta secante à essa curva nos pontos $P(0, f(0))$ e $Q(2, f(2))$ é $y-f(0)=\frac{f(2)-f(0)}{2-0}(x-0) \Rightarrow y-1=\frac{-11-1}{2} x \Rightarrow y=-6 x+1$.

e, fazendo $y=h(x)$, teremos:

$h(x)=-6 x+1$,

que é uma função polinomial e, portanto, contínua e derivável em $\mathbb{R}$.

Tomemos uma função $g(x)=f(x)-h(x)$. Essa função determina a distância vertical entre um ponto $(x, f(x))$ do gráfico de $f$ e o ponto correspondente na reta secante $\stackrel{\leftrightarrow}{P Q}$

Temos, assim:

$g(x)=f(x)-h(x) \Rightarrow g(x)=\left(3 x^{2}-12 x+1\right)-(-6 x+1) \Rightarrow g(x)=3 x^{2}-12 x+1+6 x-1$ $\Rightarrow g(x)=3 x^{2}-6 x$.

efeito,

Observemos que a função $g(x)$ cumpre as hipóteses do Teorema de Rolle. Com 
(i) $f(x)$ e $h(x)$ são contínuas em $[0,2], \operatorname{logo} g(x)$ é contínua em $[0,2]$.

(ii) $f(x)$ e $h(x)$ são deriváveis em $(0,2)$, logo $g(x)$ é derivável em $(0,2)$.

(iii) $g(0)=g(2)=0$, pois

$g(0)=3 \cdot 0^{2}-6 \cdot 0=0$ e $g(2)=3 \cdot 2^{2}-6 \cdot 2=0$.

Portanto, existe um ponto $c \in(0,2)$ tal que

$f^{\prime}(c)=\frac{f(2)-f(0)}{2-0}+3 g(c) \Rightarrow 6 c-12=\frac{-11-1}{2}+3\left(3 c^{2}-6 c\right) \Rightarrow 6 c-12=-6+9 c^{2}-18 c$

$\Rightarrow 9 c^{2}-24 c+6=0 \Rightarrow c=\frac{4 \pm \sqrt{10}}{3}$.

No entanto, devemos ter $c \in(0,2) ; \log \mathrm{O}, c=\frac{4-\sqrt{10}}{3}$.

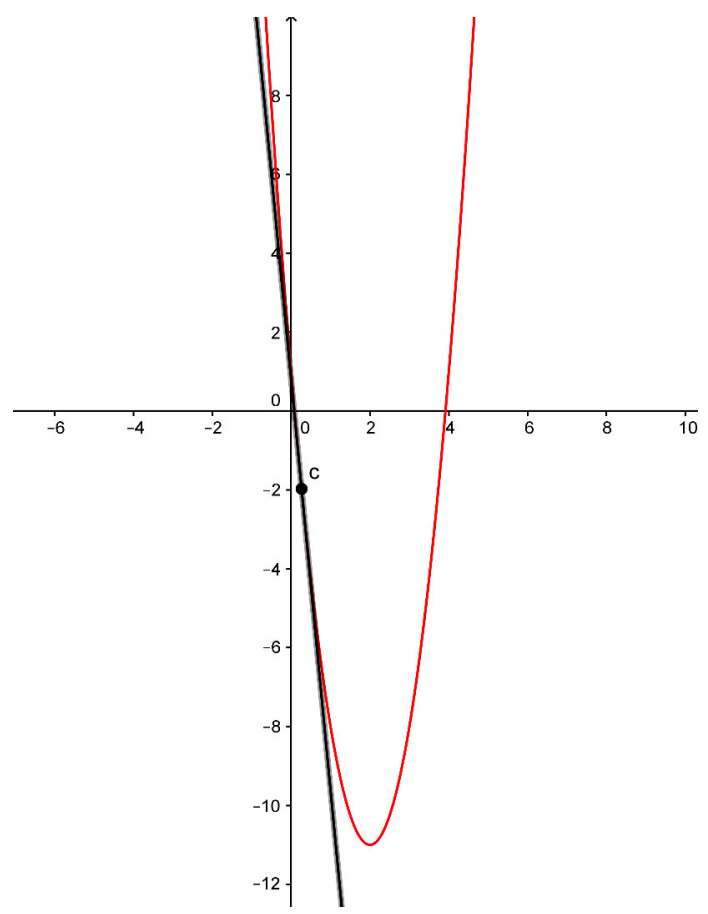

Figura 8.3: Reta tangente à curva $y=3 x^{2}-12 x+1$ no ponto $c=\frac{4-\sqrt{10}}{3}$

Exemplo: 8.4. Determine o ponto $c$ entre os pontos 0 e 2 onde a reta tangente à curva $f(x)=-2 x^{2}+8 x-3$ possui inclinação igual ao coeficiente angular da reta suporte da corda que une os pontos $P(0, f(0))$ e $Q(2, f(2))$ adicionado ao quíntuplo da distância vertical entre o ponto $(c, f(c))$ do gráfico de $f$ e o ponto correspondente na reta secante $\stackrel{\leftrightarrow}{P Q}$

Solução: 8.1.4. Por ser polinomial, temos que $f$ é contínua e derivável em $\mathbb{R}$; em particular, $f$ é contínua em $[0,2]$ e derivável em $(0,2)$. Sabendo-se que $f(0)=-3$ e $f(2)=5$, a equação da reta secante à essa curva nos pontos $P(0, f(0))$ e $Q(2, f(2))$ é

$y-f(0)=\frac{f(2)-f(0)}{2-0}(x-0) \Rightarrow y-(-3)=\frac{5-(-3)}{2} x \Rightarrow y+3=\frac{5+3}{2} x \Rightarrow y=4 x-3$.

e, fazendo $y=h(x)$, teremos:

$h(x)=4 x-3$,

que é uma função polinomial e, portanto, contínua e derivável em $\mathbb{R}$. 
Tomemos uma função $g(x)=f(x)-h(x)$. Essa função determina a distância vertical entre um ponto $(x, f(x))$ do gráfico de $f$ e o ponto correspondente na reta secante $\stackrel{\leftrightarrow}{P Q}$

Temos, assim:

$g(x)=f(x)-h(x) \Rightarrow g(x)=\left(-2 x^{2}+8 x-3\right)-(4 x-3) \Rightarrow g(x)=-2 x^{2}+8 x-3-4 x+3$ $\Rightarrow g(x)=-2 x^{2}+4 x$.

efeito,

Observemos que a função $g(x)$ cumpre as hipóteses do Teorema de Rolle. Com

(i) $f(x)$ e $h(x)$ são contínuas em $[0,2]$, logo $g(x)$ é contínua em $[0,2]$.

(ii) $f(x)$ e $h(x)$ são deriváveis em $(0,2)$, logo $g(x)$ é derivável em $(0,2)$.

(iii) $g(0)=g(2)=0$, pois

$g(0)=-2 \cdot 0^{2}+4 \cdot 0=0$

$\mathrm{e}$

$g(2)=-2 \cdot 2^{2}+4 \cdot 2=0$.

Portanto, existe um ponto $c \in(0,2)$ tal que

$f^{\prime}(c)=\frac{f(2)-f(0)}{2-0}+5 g(c) \Rightarrow-4 c+8=\frac{5-(-3)}{2}+5\left(-2 c^{2}+4 c\right) \Rightarrow-4 c+8=4-10 c^{2}+20 c$

$\Rightarrow 10 c^{2}-24 c+4=0 \Rightarrow c=\frac{12 \pm \sqrt{104}}{10}$.

No entanto, devemos ter $c \in(0,2)$; logo,

$c=\frac{12-\sqrt{104}}{10}$.

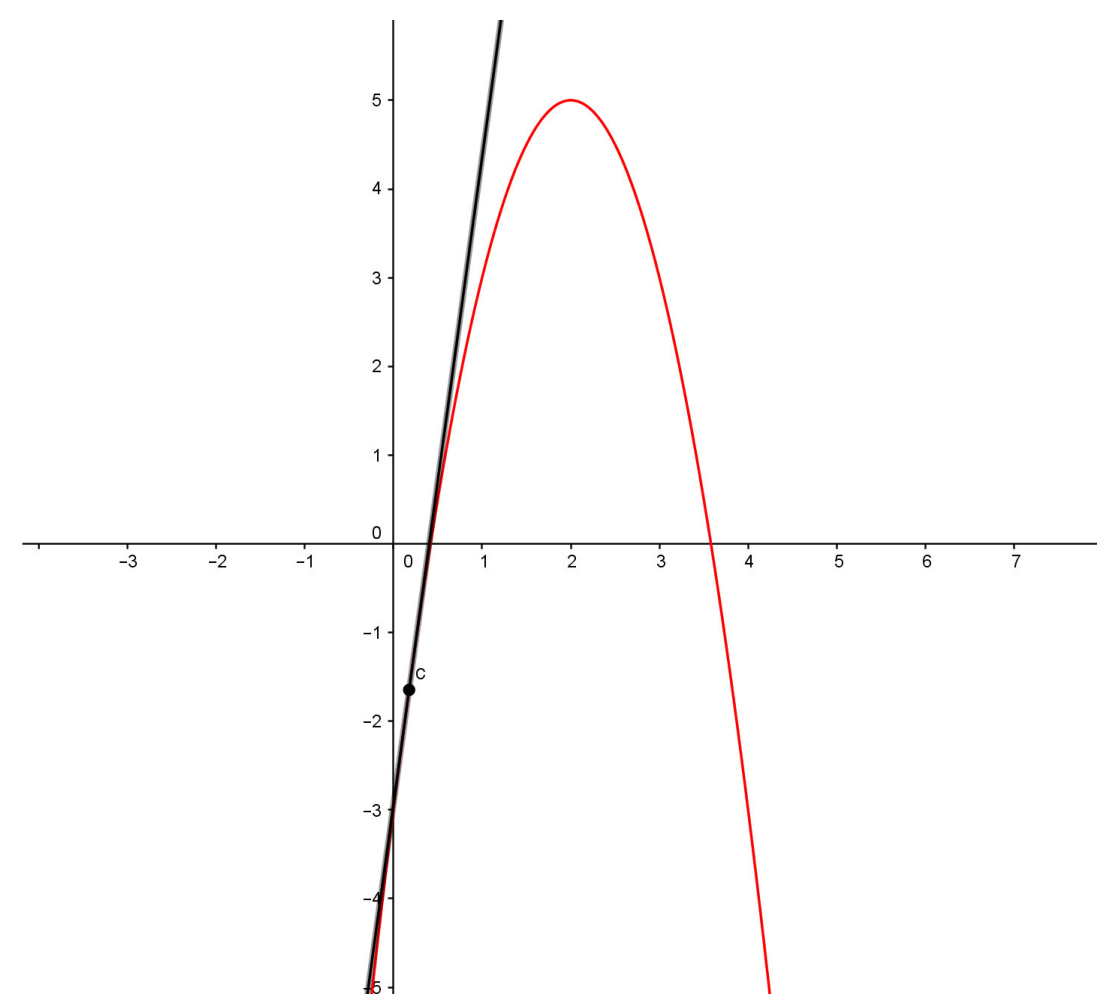

Figura 8.4: Reta tangente à curva $y=-2 x^{2}+8 x-3$ no ponto $c=\frac{12-\sqrt{104}}{10}$ 
Exemplo: 8.5. Determine o ponto $c$ entre os pontos 1 e 3 onde a reta tangente à curva $f(x)=-x^{2}+6 x-4$ possui inclinação igual ao coeficiente angular da reta suporte da corda que une os pontos $P(1, f(1))$ e $Q(3, f(3))$ adicionado à metade da distância vertical entre o ponto $(c, f(c))$ do gráfico de $f$ e o ponto correspondente na reta secante $\overleftrightarrow{P Q}$.

Solução: 8.1.5. Por ser polinomial, temos que $f$ é contínua e derivável em $\mathbb{R}$; em particular, $f$ é contínua em $[1,3]$ e derivável em $(1,3)$. Sabendo-se que $f(1)=1$ e $f(3)=5$, a equação da reta secante à essa curva nos pontos $P(1, f(1))$ e $Q(3, f(3))$ é

$y-f(1)=\frac{f(3)-f(1)}{3-1}(x-1) \Rightarrow y-1=\frac{5-1}{2}(x-1) \Rightarrow y-1=2 x-2 \Rightarrow y=2 x-1$.

e, fazendo $y=h(x)$, teremos:

$h(x)=2 x-1$, que é uma função polinomial e, portanto, contínua e derivável em $\mathbb{R}$.

Tomemos uma função $g(x)=f(x)-h(x)$. Essa função determina a distância vertical entre um ponto $(x, f(x))$ do gráfico de $f$ e o ponto correspondente na reta secante $\stackrel{\leftrightarrow}{P Q}$

Temos, assim:

$g(x)=f(x)-h(x) \Rightarrow g(x)=\left(-x^{2}+6 x-4\right)-(2 x-1) \Rightarrow g(x)=-x^{2}+6 x-4-2 x+1$ $\Rightarrow g(x)=-x^{2}+4 x-3$.

efeito,

Observemos que a função $g(x)$ cumpre as hipóteses do Teorema de Rolle. Com

(i) $f(x)$ e $h(x)$ são contínuas em $[1,3]$, logo $g(x)$ é contínua em $[1,3]$.

(ii) $f(x)$ e $h(x)$ são deriváveis em $(1,3)$, logo $g(x)$ é derivável em $(1,3)$.

(iii) $g(1)=g(3)=0$, pois

$g(1)=-(-1)^{2}+4 \cdot 1-3=0$ e $g(3)=-3^{2}+4 \cdot 3-3=0$.

Portanto, existe um ponto $c \in(1,3)$ tal que

$f^{\prime}(c)=\frac{f(3)-f(1)}{3-1}+\frac{1}{2} g(c) \Rightarrow-2 c+6=\frac{5-1}{2}+\frac{1}{2}\left(-c^{2}+4 c-3\right) \Rightarrow-2 c+6=2-\frac{c^{2}}{2}+2 c-\frac{3}{2}$

$\Rightarrow-4 c+12=4-c^{2}+4 c-3 \Rightarrow c^{2}-8 c+11=0 \Rightarrow c=\frac{8 \pm 2 \sqrt{5}}{2} \Rightarrow c=4 \pm \sqrt{5}$.

No entanto, devemos ter $c \in(1,3) ; \operatorname{logo}, c=4-\sqrt{5}$.

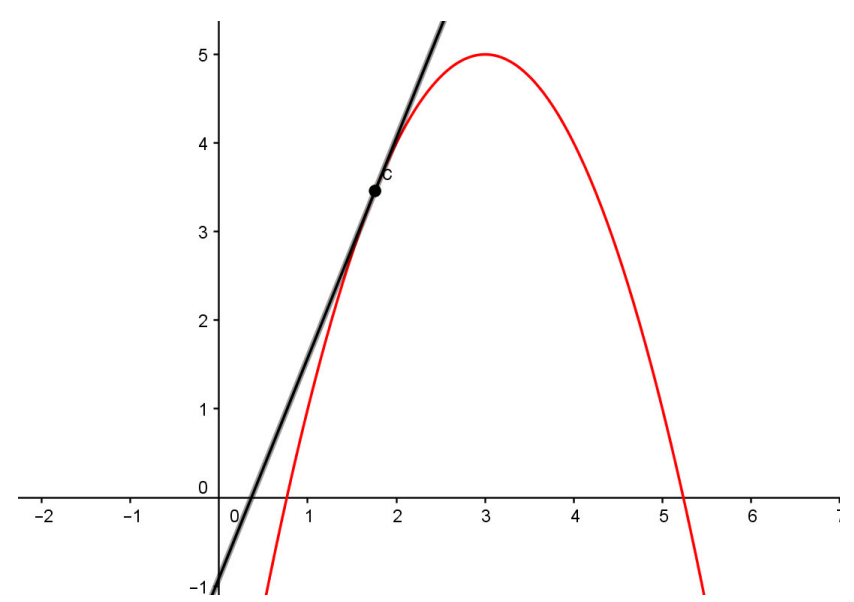

Figura 8.5: Reta tangente à curva $y=-x^{2}+6 x-4$ no ponto $c=4-\sqrt{5}$ 


\section{CONSIDERAÇÕES FINAIS}

Este trabalho apresenta uma ferramenta que permite calcular um determinado ponto de uma função contínua onde a tangente à curva tem uma inclinação específica desejada. O resultado apresentado pode ser utilizado como ferramenta para resolução de exercícios de cálculo, associado a outras resultados como os teoremas que lhe servem de base.

Todos os conceitos, propriedades e teoremas abordados nos capítulos 2, 3, 4, 5 e 6 fundamentaram nosso estudo para que pudéssemos chegar ao resultado apresentado no capítulo 8.

O Teorema 8.1.1, que é o resultado principal deste trabalho, pode ser aplicado no Ensino Médio associado ao estudo da geometria analítica.

Como sugestão para trabalhos futuros deixamos a possibilidade de estender esta aplicação particular para outros epaços métricos. 


\section{REFERÊNCIAS}

[1] Flemming, D. M.; GONÇALVES, M. B. Cálculo A: funções, limite, derivação, integração. 6. ed. Revista e ampliada. São Paulo: Pearson Prentice Hall, 2006.

[2] STEWART, J. Cálculo, volume I. 5. ed - tradução Antonio Carlos Gilli Martins. São Paulo: Cengage Learning, 2008.

[3] LIMA, E. L. Análise Real volume 1. Funções de uma variável real. 10. ed. Rio de Janeiro: Instituto Nacional de Matemática Pura e Aplicada (IMPA), 2008.

[4] LIMA, E. L. et. al. A Matemática do Ensino Médio - volume 1. 10. ed. Rio de Janeiro: Sociedade Brasileira de Matemática (SBM), 2012.

[5] GUIDORIZZI, H. L. Um Curso de Cálculo - volume 1. 5. ed. Rio de Janeiro: LTC, 2008.

[6] MUNIZ NETO, A. C. Tópicos de Matemática Elementar - volume 3. introdução à análise. 2. ed. Rio de Janeiro: Sociedade Brasileira de Matemática (SBM), 2013.

[7] ÁvILA, G. S. de Souza. Introdução à análise matemática. 2. ed. rev. São Paulo: Edgard Blücher, 1999.

[8] MUNIZ NETO, A. C. Fundamentos de Cálculo. 1. ed. Rio de Janeiro: Sociedade Brasileira de Matemática (SBM), 2015.

[9] Uma aplicação interessante do teorema de Rolle. In: Problemas e Teoremas. 2009. Disponível em: <https://problemasteoremas.wordpress.com/2009/05/09/uma-aplicacaointeressante-do-teorema-de-rolle/>. Acesso em: 29 mar. 2016. 